\title{
The ALMA Lupus protoplanetary disk survey: evidence for compact gas disks and molecular rings from $\mathrm{CN}$
}

\author{
S. E. van Terwisga ${ }^{1}$, E. F. van Dishoeck ${ }^{1,2}$, P. Cazzoletti ${ }^{2}$, S. Facchini ${ }^{8}$, L. Trapman ${ }^{1}$, J. P. Williams ${ }^{3}$, C. F. Manara ${ }^{8}$, \\ A. Miotello ${ }^{8}$, N. van der Marel ${ }^{3,4}$, M. Ansdell ${ }^{3,5}$, M. R. Hogerheijde ${ }^{1,7}$, M. Tazzari ${ }^{6}$, and L. Testi ${ }^{8,9}$ \\ ${ }^{1}$ Leiden Observatory, Leiden University, PO Box 9513, 2300 RA Leiden, The Netherlands \\ e-mail: terwisga@strw.leidenuniv.nl \\ ${ }^{2}$ Max-Planck-Institut für Extraterrestrische Physik, Gießenbachstraße, 85741 Garching bei München, Germany \\ ${ }^{3}$ Institute for Astronomy, University of Hawai ‘i at Mānoa, 2680 Woodlawn Dr., Honolulu, HI, USA \\ ${ }^{4}$ Herzberg Astronomy \& Astrophysics Programs, NRC of Canada, 5017 West Saanich Road, Victoria, BC V9E 2E7, Canada \\ ${ }^{5}$ Department of Astronomy, University of California, Berkeley, CA 94720, USA \\ ${ }^{6}$ Institute of Astronomy, University of Cambridge, Madingley Road, CB3 OHA, Cambridge, UK \\ 7 Anton Pannekoek Institute for Astronomy, University of Amsterdam, Postbus 94249, 1090 GE Amsterdam, The Netherlands \\ ${ }^{8}$ European Southern Observatory, Karl-Schwarzschild-Str. 2, 85748 Garching bei München, Germany \\ ${ }^{9}$ INAF - Osservatorio Astrofisico di Arcetri, Largo E. Fermi 5, 50125 Firenze, Italy
}

Received 17 September 2018 / Accepted 6 November 2018

\begin{abstract}
Context. The cyanide radical $\mathrm{CN}$ is abundant in protoplanetary disks, with line fluxes often comparable to those of ${ }^{13} \mathrm{CO}$. It is known to be sensitive to UV irradiation of the upper disk atmosphere, with models predicting ring-shaped emission.

Aims. We seek to characterize the $\mathrm{CN}$ emission from 94 Class-II disks in the Lupus star-forming region, compare it to observations in other regions, and interpret our observations with a grid of models. The $\mathrm{CN}$ emission morphology is discussed for two primordial disks, $\mathrm{Sz} 71$ and $\mathrm{Sz} 98$, and is modeled in more detail.

Methods. ALMA observed CN $N=3-2$ in Lupus disks down to sensitivities better than previous surveys. Models constructed with the physico-chemical code DALI are used to study the integrated fluxes of the disks and resolved emission of CN in disks without (dust) substructures.

Results. CN $N=3-2$ is bright, and detected in $38 \%$ of sources, but its disk-integrated flux is not strongly correlated to either ${ }^{13} \mathrm{CO}$ or continuum flux. Compared to pre-ALMA single-dish surveys, no significant difference in the CN flux distributions in Lupus and Taurus-Auriga is found, although $\rho$ Ophiuchus disks may be fainter on average. We find ring-shaped CN emission with peak radii of $\sim 50 \mathrm{AU}$ in two resolved disks.

Conclusions. A large fraction of sources are faint in $\mathrm{CN}$; only exponential gas surface density cutoffs at $R_{\mathrm{c}} \leq 15 \mathrm{AU}$ can reconcile observations with models. This is the first observational evidence of such a compact gas disk population in Lupus. Absolute intensities and the emission morphology of $\mathrm{CN}$ are reproduced by DALI models without the need for any continuum substructure; they are unrelated to the $\mathrm{CO}$ snowline location. The observations presented here, together with the modeling of these rings, provide a new probe of the structure and conditions in disks, and particularly their incident UV radiation field, if disk size is determined from the data.
\end{abstract}

Key words. astrochemistry - protoplanetary disks - stars: pre-main sequence - stars: individual: Sz 98 - stars: individual: Sz 71 techniques: interferometric

\section{Introduction}

The primary motivation for the study of protoplanetary disks is to understand the formation of planetary systems. For this purpose it is essential to characterize the structure and composition of both dust and gas in the disks, with different molecules providing different information on the gas structure and conditions.

Initially, most disk observations were limited by low spatial resolution and sensitivity, and focused on disk-integrated observations of well-known, bright objects (see the review by Henning \& Semenov 2013). In recent years, ALMA has been instrumental in detecting as well as resolving millimeter(mm)sized dust and a number of gas species near the midplanes of protoplanetary disks, thus providing a new window on the environment where planets are formed. A number of large, unbiased, and deep surveys have significantly improved our understanding of the protoplanetary disk populations in nearby star-forming regions (e.g., Andrews \& Williams 2005; Pascucci et al. 2016; Barenfeld et al. 2016; Ansdell et al. 2016). Among the puzzling results from these surveys is the weaker-than-expected $\mathrm{CO}$ isotopolog emission when assuming normal $\mathrm{CO}$ abundances and an interstellar medium (ISM)-like gas-to-dust ratio (Ansdell et al. 2016; Miotello et al. 2017; Long et al. 2017).

While most of these surveys focused exclusively on the (resolved) continuum emission of Class-II disks, or on CO and its isotopologs, relatively few surveys have targeted other molecular species. Targeting other molecules is scientifically valuable: if their chemistry is sufficiently well understood, they can provide additional independent constraints on the properties of disks. However, molecular lines are often faint, or the chemical pathways behind their formation and destruction not well understood, making them less attractive targets for a survey.

The cyanide radical $(\mathrm{CN})$ is, in this context, of particular interest. After ${ }^{12} \mathrm{CO}$, it is one of the brightest molecules in 
disks, and has line fluxes comparable to or higher than those of ${ }^{13}$ CO (e.g., Dutrey et al. 1997; Thi et al. 2004; Öberg et al. 2011; Salter et al. 2011). Due to its brightness, several large single-dish surveys of this molecule have already been performed using the IRAM 30-m telescope (Guilloteau et al. 2013; Reboussin et al. 2015). Together these surveys cover 74 disks in Taurus and $\rho$ Ophiuchi. However, the samples studied suffer from two key drawbacks: they are not complete, and are biased towards the brightest or most radially extended sources at $\mathrm{mm}$ wavelengths.

Despite these issues, the abundance of $\mathrm{CN}$ has been suggested to be enhanced in Class-II versus Class-I disks (Kastner et al. 2014; Guilloteau et al. 2016). Also, Guilloteau et al. (2014) successfully used the cyanide radical as a stellar mass probe. However, in order to get a better understanding of the dependence of $\mathrm{CN}$ on disk properties, it is necessary to use an unbiased, large, and uniformly observed sample of disk observations.

The observational basis for this study is the complete survey of Class-II disks in the Lupus star-forming region (Ansdell et al. 2016, 2018). This survey offers good angular resolution at $\sim 0.3^{\prime \prime}$, or a $\sim 24 \mathrm{AU}$ radius at $160 \mathrm{pc}$ (the typical distance for these objects, based on Gaia DR2; Gaia Collaboration 2018; Bailer-Jones et al. 2018). The sensitivity of this dataset is a factor of approximately three better than previous large single-dish CN surveys. Moreover, the sample studied here is unbiased. By covering 94 of 95 Class-II disks in Lupus I, III, and IV with stellar masses $\geq 0.1 M_{\odot}$, this survey is very complete and more than doubles the total number of disks observed in $\mathrm{CN}$.

From an evolutionary perspective, the objects in the Lupus survey must be relatively close in age, around $1-3 \mathrm{Myr}$ (Comerón 2008), and formed in a similar environment. This reduces the impact of different disk ages on observational properties. Finally, the Lupus disks have been characterized at multiple wavelengths. Apart from the dust continuum, the Lupus disk survey targeted ${ }^{12} \mathrm{CO}$ and its most common isotopologs, ${ }^{13} \mathrm{CO}$ and $\mathrm{C}^{18} \mathrm{O}$. Additionally, intermediate-resolution X-shooter spectra of most sources in the sample have also been taken (Alcalá et al. 2014, 2017), meaning that accurate measurements of the host star properties and accretion rates are available.

Apart from providing disk-integrated observations of $\mathrm{CN}$ towards many sources, resolved images are also obtained, even at integration times of just one minute. In comparison to preALMA interferometric studies of individual objects (e.g., Öberg et al. 2011; Guilloteau et al. 2014), our effective physical resolution is improved by a factor of $\geq 2$. With these short integration times, the signal-to-noise ratio $(\mathrm{S} / \mathrm{N})$ of the molecular gas emission is low: for the brightest sources in $\mathrm{CN}$, the line peak has a $S / N \approx 12$ relative to the channel noise. However, we are able to discuss in greater detail the ring-like radial distribution of $\mathrm{CN}$ emission in two favorably inclined, bright sources, and study its underlying causes in a general way.

This is relevant because, in recent years, the high-resolution capabilities of ALMA have lead to the discovery of many rings and other radial structures, in gas emission (e.g., Kastner et al. 2015; Teague et al. 2017) and in dust (e.g., ALMA Partnership 2015; Isella et al. 2016), in a variety of sources and molecules. These structures occur both in transitional disks, which are characterized by central cavities in their dust distribution (Espaillat et al. 2014; van der Marel et al. 2016a) as well as in full disks, which are the focus of this article. Indeed, ALMA observations of TW Hya also show CN to be distributed in a ring (Teague et al. 2016), suggesting this type of emission morphology may be more common for this species.
For molecular species with ring-like emission, several different processes have been identified as their root cause: $\mathrm{CO}$ is depleted in at least some of the gaps in the dust in the HD 163296 system (Isella et al. 2016), while CS emission coincides with a region of decreased dust surface density in TW Hya (Teague et al. 2017). More complex chemical processes also play a role for some species: dust grains locking up volatile carbon and oxygen in the outer disk have been suggested as an explanation for rings observed in $\mathrm{C}_{2} \mathrm{H}$ and $c-\mathrm{C}_{3} \mathrm{H}_{2}$ (Kastner et al. 2015; Bergin et al. 2016). Snow lines are also an important mechanism for causing the ring-like emission of some molecules. For instance, $\mathrm{N}_{2} \mathrm{H}^{+}$is distributed in a ring in several disks (Qi et al. 2013, 2015), of which the radius is sensitive to the position of the CO ice line (Aikawa et al. 2015; van't Hoff et al. 2017). Many other molecular species are now showing a rich variety of emission morphologies, including single and multiple rings: $\mathrm{DCO}^{+}$, DCN, $\mathrm{H}^{13} \mathrm{CN}$, and $\mathrm{H}^{13} \mathrm{CO}^{+}, \mathrm{H}_{2} \mathrm{CO}$ (Mathews et al. 2013; Öberg et al. 2015; Huang et al. 2017; Carney et al. 2017; Salinas et al. 2017). However, for many of these species, finding the underlying cause of the emission morphology from observations alone is difficult, due to the variety in emission profiles between different molecules, and between disks.

To study integrated fluxes and the radial distribution of $\mathrm{CN}$ emission in disks, the chemical network that sets its formation and destruction must be understood. Significant advances have recently been made on this front: the physical-chemical code DALI (Bruderer et al. 2012; Bruderer 2013), which selfconsistently calculates the chemical and thermal structure of the disk models, has been expanded to include nitrogen chemistry (Visser et al. 2018) using the most recent rates and branching ratios from Loison et al. (2014). Moreover, DALI now uses updated UV cross-sections for $\mathrm{CN}$ photodissociation (Heays et al. 2017). This allows us to predict the $\mathrm{CN}$ emission expected for a wide variety of disks, and study how the models depend on various disk parameters, such as radius, mass, and incident UV flux (Cazzoletti et al. 2018). Several key predictions have been found: first, an increasing integrated $\mathrm{CN}$ flux with increasing disk size; second, a strong dependence of $\mathrm{CN}$ flux on the UV radiation field of stars; and third, a relatively weak dependence on total disk mass and on the volatile carbon abundance. In terms of the distribution of $\mathrm{CN}$ emission over the disk, a ringlike morphology is always found in the models, with the ring radius depending on the characteristic radius $R_{\mathrm{c}}$ of the gas disk, the vertical profile of the disk, and the UV radiation field.

In this paper, we discuss the $\mathrm{CN}$ emission in the full Lupus Class-II disk population (Sect. 3), investigate possible correlations with other disk tracers, and compare the Lupus disks to previous large single-dish surveys. In Sect. 3.3, the properties of the full disk sample are compared to a DALI model grid, focusing on the consequences for disk gas radii and the sensitivity to UV radiation. To investigate resolved $\mathrm{CN}$ emission, two full (i.e., nontransitional) disks are described in detail (Sect. 4). These disks - Sz 98 and Sz 71 - are amongst the brightest sources in $\mathrm{CN}$, have clearly detected and resolved emission in both lines and continuum, and are not strongly inclined (around $45^{\circ}$ ). Section 4.2 discusses individual DALI models for $\mathrm{CN}$ emission in $\mathrm{Sz} 98$ and $\mathrm{Sz} 71$ to see if a smooth power-law surface-density distribution can reproduce their $\mathrm{CN}$ intensity profiles.

\section{Observations and data reduction}

The data for this project are part of the ALMA Lupus disk survey (Ansdell et al. 2016, 2018), which targeted 86 Class-II stars with ALMA Band 7 and 6 (ID: 2013.1.00220.S, 2015.1.00222.S, 
PI: J. Williams), combined with the 7 sources from the Lupus Completion Survey (ID: 2016.1.01239.S, PI: S. van Terwisga). To these 93 disks, we add previously published data for IM Lup (Öberg et al. 2011). On top of the ${ }^{12} \mathrm{CO} J=2-1,{ }^{13} \mathrm{CO}$, and $\mathrm{C}^{18} \mathrm{O} J=2-1$ and $J=3-2$ lines, and 335 and $225 \mathrm{GHz}$ continuum, the spectral settings of these surveys targeted the $\mathrm{CN} v=0$ $N=3-2$ transition in Band 7. Of the fine-structure transitions of this molecule, we focus on the brightest $(J=7 / 2-5 / 2)$ line, and in particular the $F=7 / 2-5 / 2$ and $F=9 / 2-7 / 2$ transitions at $340.247770 \mathrm{GHz}$ and the partially overlapping $F=5 / 2-3 / 2$ transition; all others are too faint to be detected in our data but these transitions together are responsible for $95 \%$ of the flux in the $J=7 / 2-5 / 2$ line in the optically thin case (Hily-Blant et al. 2017). CN was observed at a lower spectral resolution of $0.24 \mathrm{MHz}$ compared to ${ }^{13} \mathrm{CO} J=3-2$, for which a $0.12 \mathrm{MHz}$ resolution was used. The analysis in this article mainly focuses on the Band 7 results, and only uses the Band $6{ }^{12} \mathrm{CO} J=2-1$ data.

In Band 7, all M-type stars were observed for 1 min on source, while earlier-type stars were observed for $30 \mathrm{~s}$, giving continuum sensitivities of 0.25 and $0.41 \mathrm{mJy} \mathrm{beam}^{-1}$. Band 6 integration times were twice as long. For the sources in the Lupus Completion Survey, longer integration times were used, of 3.5 and $4.5 \mathrm{~min}$ in Bands 6 and 7, respectively, but a smaller beam lead to similar sensitivities (Ansdell et al. 2018); otherwise all observing parameters were identical.

Pipeline calibration of the data was performed by the NRAO. Flux, phase, bandpass and gain were calibrated using observations of Titan, J1427-4206, and J1604-4228, and J1610-3958, respectively. We also performed self-calibration on the data in order to maximize the $\mathrm{S} / \mathrm{N}$ of the resulting images. Phase- and gain self-calibration were performed for all objects at the largest solution interval of one minute (for the highest sensitivity). These disks are not so bright as to be dominated by phase- and gain noise even in the continuum images, but this procedure resulted in an improvement in $\mathrm{S} / \mathrm{N}$.

Both continuum and line imaging were performed using the clean task in CASA. The continuum of the sources discussed in detail here was imaged using Briggs weighting with a robust parameter of +0.5 after averaging over all continuum channels, which optimized our resolution and $\mathrm{S} / \mathrm{N}$ in the image. The resulting effective beam size in Band 7 is $0.3^{\prime \prime} \times 0.3^{\prime \prime}$, while in Band 6 it is $0.27^{\prime \prime} \times 0.27^{\prime \prime}$. In the Lupus completion survey, the beams were $0.25^{\prime \prime} \times 0.22^{\prime \prime}$ (Band 6) and $0.19^{\prime \prime} \times 0.18^{\prime \prime}($ Band 7), respectively.

The CN channels were binned for an effective velocity resolution of $0.5 \mathrm{~km} \mathrm{~s}^{-1}$, and imaged using natural weighting to maximize $\mathrm{S} / \mathrm{N}$. The resulting typical beam shape is $0.37^{\prime \prime} \times$ $0.31^{\prime \prime}$; for the Lupus completion survey it is $0.22^{\prime \prime} \times 0.20^{\prime \prime}$. The ${ }^{12} \mathrm{CO} J=2-1$ and ${ }^{13} \mathrm{CO} J=3-2$ lines were imaged with the same parameters. In these observations, the typical resulting channel rms of these $\mathrm{CN}$ observations is $10 \mathrm{mJy}^{\text {beam }}{ }^{-1}$. The median noise level on the disk- and velocity-integrated line fluxes is $48 \mathrm{mJy} \mathrm{km} \mathrm{s}^{-1}$.

Of the 93 disks observed with ALMA, we focus specifically on the two brightest disks that are moderately inclined $\left(<50^{\circ}\right)$, resolved across multiple beams, have high-S/N momentzero maps, and do not show any signs of depleted inner dust cavities. For these two disks, $\mathrm{Sz} 98$ and $\mathrm{Sz} 71$, it is possible to study the spatial distribution of $\mathrm{CN}$. To generate and analyze the moment-zero maps at the highest sensitivity without introducing bias (by, e.g., using significance cuts in the individual channels), image-plane Keplerian masking was used to create their moment-zero maps (Loomis et al. 2018; Salinas et al. 2017).
For this method to work, several properties of the system must be known. The stellar masses have been derived by determining the stellar properties of the targets with optical spectroscopy (Alcalá et al. 2017) and comparing the stellar temperature and luminosity with evolutionary models by Siess et al. (2000), while the $\mathrm{mm}$-continuum observations allowed us to constrain the position angles and inclinations of the disk using the imfit task in CASA, version 4.3.1. These results are combined with a line-width uncertainty factor (here taken as $0.5 \mathrm{~km} \mathrm{~s}^{-1}$ for $\mathrm{CN}$, and $0.3 \mathrm{~km} \mathrm{~s}^{-1}$ for the $\mathrm{CO}$ isotopolog lines) which accounts for uncertainties in the precise stellar position, the disk position angle and inclination, and the effect of adjacent hyperfine lines in CN (specifically, the partial overlap of the $F=7 / 2-5 / 2$ and $F=9 / 2-7 / 2$ transition with the $F=5 / 2-3 / 2$ transition). The resulting mask allowed us to use only those voxels in the objects' data cubes in which a signal can be reasonably expected. Compared to a normal moment-zero map, the biggest gain in sensitivity using this mask is in the outer regions of the disk, where (often) only a single channel contains a line signal. For ${ }^{13} \mathrm{CO}$ in $\mathrm{Sz} 98$, we additionally used $u v$-plane tapering to create a $0.5^{\prime \prime}$ circular beam, in order to maximize $\mathrm{S} / \mathrm{N}$.

\section{Integrated CN flux survey results}

The full Lupus sample consists of 95 Class-II disks, 93 of which have been observed in $\mathrm{CN}$ in this survey. Of the two missing objects, Sz 91 and IM Lup, only Sz 91 has no CN observations available. Instead of the $\mathrm{CN} N=3-2$ transition, $\mathrm{CN} N=2-1$ has been observed and detected towards IM Lup by Öberg et al. (2011).

In these observations, 36 of 94 sources are detected, for a total detection rate of $38 \%$ across the sample. All but three of these sources are also detected in ${ }^{13} \mathrm{CO}$. The brightest sources in terms of CN flux are the transition disk J16083070-3828268 (van der Marel et al. 2018), Sz 98, V1094 Sco (van Terwisga et al. 2018), IM Lup, Sz 133, and Sz 71. Interestingly, the transition disks with large inner cavities in the sample that were identified in van der Marel et al. (2018) are all detected in CN. These sources were also found to be among the brightest in the sample in continuum and ${ }^{13} \mathrm{CO}$ emission. Integrated fluxes for these objects were determined in the same way as those in Ansdell et al. $(2016,2018)$ : circular aperture photometry was performed on the source position after integrating over all channels with significant emission, with the smallest aperture containing the full disk flux determined using a curve-of-growth method. For nondetections, a beam-sized aperture was used, and a conservative velocity range with a width of $10 \mathrm{~km} \mathrm{~s}^{-1}$, to be consistent with our previously used noise definitions.

\section{1. $\mathrm{CN}$ versus other disk tracers: ${ }^{13} \mathrm{CO}$ and continuum}

The large, unbiased sample of Class-II disks in Lupus allows us to compare the resulting $\mathrm{CN}$ fluxes to the other important disk tracers: their continuum flux (proportional to the disk dust mass, if we assume the emission to be optically thin at these wavelengths) and integrated ${ }^{13} \mathrm{CO} J=3-2$ emission, which is the most commonly detected $\mathrm{CO}$ isotopolog, and more optically thin than ${ }^{12} \mathrm{CO} J=2-1$. The results of this comparison are presented in Fig. 1. Appendix A shows $\mathrm{CN}$ versus the $\mathrm{C}^{18} \mathrm{O}$ emission in Fig. A.1, but we do not discuss it further here due to the low detection rate of this molecule in both Band 6 and Band 7.

The CN fluxes detected towards Lupus span several orders of magnitude, and are presented in full in Table E.1. Compared to the continuum- and ${ }^{13} \mathrm{CO}$ fluxes, a large scatter becomes 

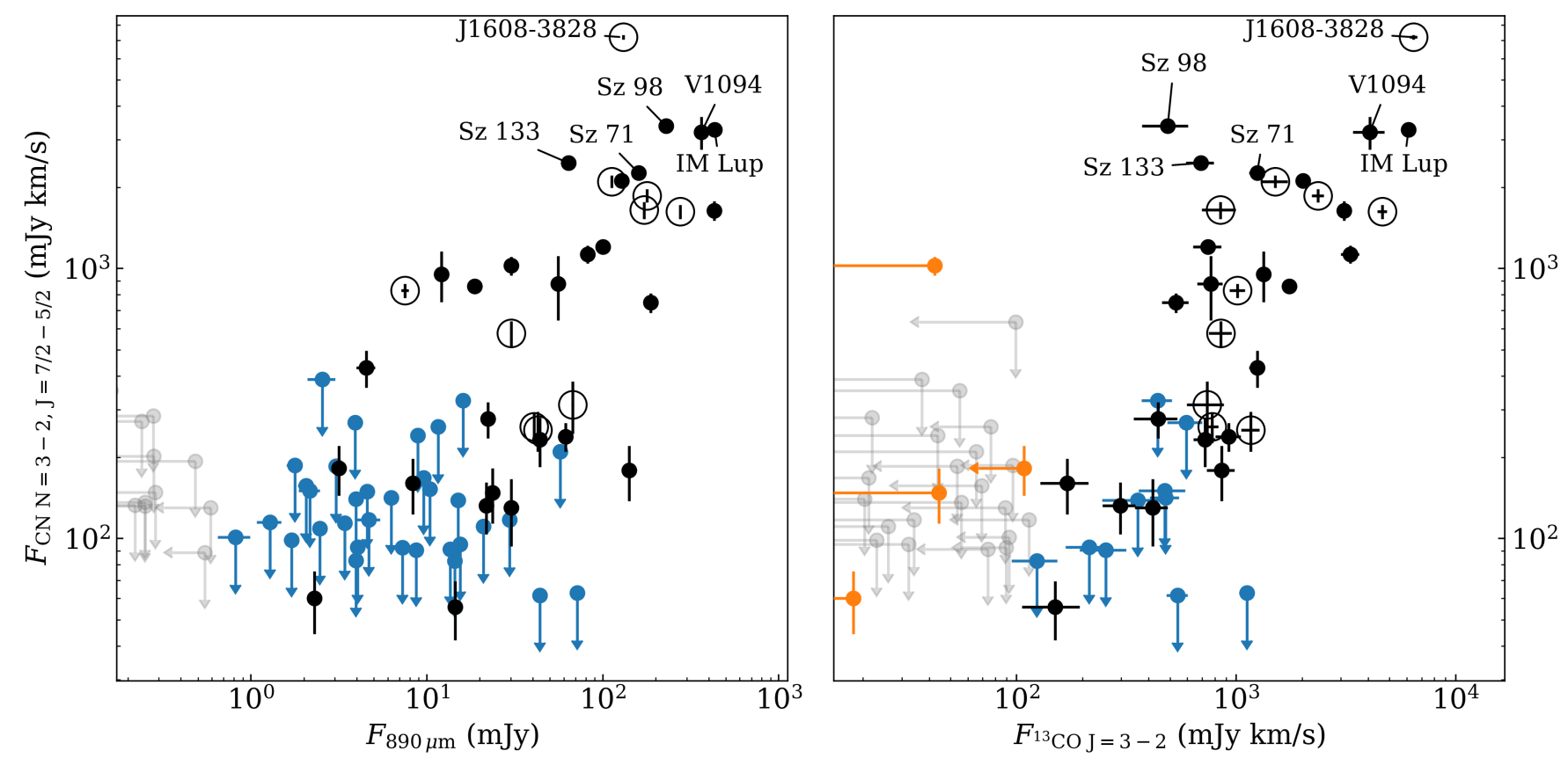

Fig. 1. Disk-integrated CN $N=3-2, J=7 / 2-5 / 2$ vs. Band 7 continuum (left panel) and integrated ${ }^{13} \mathrm{CO} J=3-2$ fluxes (right panel) for the Lupus disk sample. Detected sources are black, $\mathrm{CN}$ upper limits are blue, ${ }^{13} \mathrm{CO}$ upper limits orange, and double upper limits are gray. Transition disks are marked with a circle. All upper limits are at the $3 \sigma$ level. The six brightest disks are labeled.

apparent, of several orders of magnitude, especially due to the impact of upper limits in the data. Consider, for example, the position of Sz 71 (labeled) in both panels of Fig. 1: a source with similarly bright ${ }^{13} \mathrm{CO}$ is undetected in $\mathrm{CN}$, while another disk with a similar $890 \mu \mathrm{m}$ flux has $\mathrm{CN}$ emission that is an order of magnitude fainter. Similar behavior is seen in the other direction.

To formally test for correlations between these observables, a modified Kendall-rank test taking into account upper limits was used (Isobe et al. 1986). This test confirms that neither the $890 \mu \mathrm{m}$ continuum nor the ${ }^{13} \mathrm{CO} J=3-2$ line flux are correlated with the $\mathrm{CN}$ flux $(p>0.05)$, consistent with the results from the IRAM 30-m telescope survey in Taurus-Auriga by Guilloteau et al. (2013). Not being able to reject the null result does not mean that no information can be extracted from this figure; however, to do so, more sophisticated models are needed, which are discussed in Sect. 3.3.

\section{2. $C N$ in Lupus versus Taurus-Auriga and $\rho$ Ophiuchi}

Comparing the results of the Lupus sample to those of other literature surveys allows us to test if different regions (possibly with different ages, typical disk sizes, or external UV fields) have different $\mathrm{CN}$ emission properties. Here, we compare the Lupus $\mathrm{CN}$ observations to the results presented for $\mathrm{CN}$ in Guilloteau et al. (2013) and Reboussin et al. (2015), who targeted 42 and 30 stars respectively with the IRAM 30-m single-dish telescope, primarily in the Taurus-Auriga and $\rho$ Oph star-forming regions.

Some caveats apply to this analysis. First, the IRAM 30-m sample covers a range of spectral types, stellar luminosities, and disk masses, but might be biased to bright or radially extended targets. Large-scale cloud contamination is a possibility, but is ruled out by the authors of both studies. Likewise, we must assume similar underlying stellar properties in all these samples. Fortunately, this assumption has already been found to hold for these regions (Ansdell et al. 2016; Cieza et al. 2019), and we do not expect this to have a significant effect.
Second, previous $\mathrm{CN}$ observations have typically targeted the $N=2-1$ lines. Specifically, both Guilloteau et al. (2013) and Reboussin et al. (2015) quote integrated fluxes for the $N=2-1, J=5 / 2-3 / 2$ components, which need to be related to the transition observed here. The $\mathrm{CN}(N=2-1$, $J=5 / 2-3 / 2) /(N=3-2, \quad J=7 / 2-5 / 2)$-ratio is partially dependent on the disk (temperature) structure. Based on the grid of models discussed in Cazzoletti et al. (2018), this ratio is found to vary between 1.1 and 2.1, depending on the disk flaring angle and scale height, but only weakly on mass. For IM Lup, a detailed model of the disk structure exists, based on both gas and dust observations (Cleeves et al. 2016). For this disk, with flaring angle $\psi=0.15$ and scale height $h_{\mathrm{c}}=0.12$, we can infer a scaling ratio of 1.4 , and an $N=3-2, J=7 / 2-5 / 2$ flux of $1.4 \pm 0.1 \mathrm{Jy} \mathrm{km} \mathrm{s}^{-1}$.

Since such detailed models are not generally available, a single scaling factor was used here to obtain estimated $\mathrm{CN}$ $N=3-2, \quad J=7 / 2-5 / 2$ fluxes from these surveys, assuming an intermediate value of 1.6. Where appropriate, the typical spread of values introduced by this assumption is indicated in the figures by arrows to either side. It is important to note that - while some transition disks are included - these were not included in the models used to derive the flux ratios used here. However, transition disks with large inner cavities are rare enough (making up $\sim 11 \%$ of the Lupus disks; van der Marel et al. 2018) that we do not expect this to significantly affect our results.

The results of three different $\mathrm{CN}$ surveys are shown in Fig. 2. Using a logrank test (applicable to unbiased data with upper limits), we cannot identify a significant difference in the $\mathrm{CN}$ flux distribution between the disks in Lupus (this survey) and Taurus-Auriga (Guilloteau et al. 2013; $p=0.064$ ) if we use $p=0.05$ as the rejection criterium, and the difference becomes significant only if we take an $(N=2-1, J=$ $5 / 2-3 / 2) /(N=3-2, \quad J=7 / 2-5 / 2)$ flux ratio of 1.1 . TaurusAuriga seems to be relatively richer in intermediate-luminosity disks, which drive most of this trend. It is however possible that 


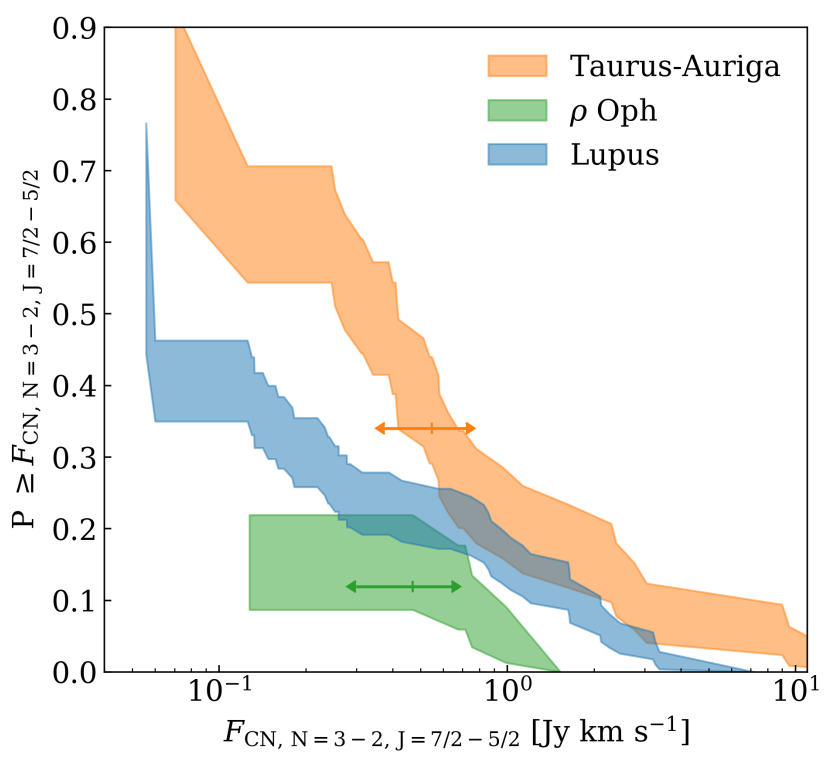

Fig. 2. Disk-integrated CN $N=3-2, \quad J=7 / 2-5 / 2$ fluxes in the complete Lupus disk survey (this work) compared to those of a sample of disks in Taurus-Auriga (Guilloteau et al. 2013) and $\rho$ Oph (Reboussin et al. 2015). Source distances for all objects were taken from Gaia DR2, if available; otherwise, median distances to the three populations were used (Gaia Collaboration 2018; Bailer-Jones et al. 2018). Fluxes were then scaled to the median distance of Lupus $(160 \mathrm{pc})$. Colored arrows indicate the effect of using different $\mathrm{CN}$ $(N=2-1, J=5 / 2-3 / 2) /(N=3-2, J=7 / 2-5 / 2)$ scaling parameters over the full range of model values.

the disks sampled in the Guilloteau et al. (2013) survey are biased towards those disks in which both continuum and lines are more readily detected, leading to apparently brighter disks in $\mathrm{CN}$. This seems to be the case for the $\rho$ Ophiuchus sample; comparing it to the resolved ALMA images in Cieza et al. (2019) reveals a trend towards more radially extended disks, with only two objects included in the $\mathrm{CN}$ survey being unresolved. In Taurus-Auriga, such a bias would lead to the difference with Lupus becoming less significant, especially at lower $\mathrm{CN}$ luminosities. Comparing the Lupus $\mathrm{CN}$ results to those of the $\rho$ Oph disk sample presented in Reboussin et al. (2015), however, the difference in flux distributions is always significant: $\rho$ Oph has fainter overall $\mathrm{CN}$ emission, and a lower detection rate. A bias towards more radially extended disks in this region would make the lack of $\mathrm{CN}$ emission more significant, since we would expect such disks to be brighter rather than fainter on average (see Sect. 3.3.1, below).

\section{3. $C N$ models in the context of the Lupus population}

The Lupus disk survey is uniquely suited to testing models of $\mathrm{CN}$ production in protoplanetary disks, both by looking at individual resolved objects and by comparing the full sample properties to the results from a large grid of models. In this section, this model grid is generated by the DALI physical-chemical disk code, which produces chemical abundances, performs non-local thermal equilibrium (LTE) excitation calculations, and produces ray-traced images. As mentioned, Cazzoletti et al. (2018) find several key results for $\mathrm{CN}$ emission in disks in their models made with this version of the code: first, an increasing integrated $\mathrm{CN}$ flux with increasing disk size; second, a strong dependence of flux on the UV radiation field of stars; and third, a relatively weak dependence on total disk mass or volatile carbon abundance.
Here, a small DALI grid is used for comparison with our data, and the results of two previous surveys by Guilloteau et al. (2013) and Reboussin et al. (2015). Since both the Lupus data and the previous surveys give fine-structure fluxes, we used the collisional rates for $\mathrm{CN}$ from Lique et al. (2010), and raytrace the $N=3-2, J=7 / 2-5 / 2$ and $N=2-1, J=5 / 2-3 / 2$ lines. The grid is constructed using the standard viscous disk model of Lynden-Bell \& Pringle (1974) as used, for example, in Andrews et al. (2011), and samples disks with critical radii $R_{\mathrm{c}}$ in the gas disk between 15-60 AU, and in the range $2 \times 10^{-6}-9 \times$ $10^{-2} M_{\odot}$ in total disk mass. The stellar spectrum is given by a $4000-\mathrm{K}$ black-body spectrum emitted by a star of $1.65 R_{\odot}$; the grid includes models with UV excess (spanning two orders of magnitude in flux) as well as models without excess UV added to this stellar spectrum. The disk structure does not vary in these models: the surface density parameter $\gamma=1$, while the scale height at $60 \mathrm{AU} h_{\mathrm{c}}=0.1$ and the flaring angle $\psi=0.2$. While the $\mathrm{CN}$ flux is sensitive to these parameters, their effect is smaller than that of the varying UV luminosity used in these models (Cazzoletti et al. 2018), especially since the variation in flaring angles seems to be fairly small (Bustamante et al. 2015). Although our disks are not in hydrostatic equilibrium, the range of scale heights used here generally reproduces the observed SEDs of disks (van der Marel et al. 2016b).

In order to compare the models to the observational data, several assumptions must be made. First, a disk (gas) mass tracer is needed. Since ${ }^{13} \mathrm{CO}$ is not be a good tracer of the total gas mass, we will assume that the gas-to-dust ratio of these disks is 100 throughout, and that the dust is optically thin, leading to the same approximation used in Ansdell et al. (2016):

$M_{\text {disk }}=100 \frac{F_{890 \mu \mathrm{m}} d^{2}}{\kappa_{v} B_{v}\left(T_{\text {dust }}\right)}$,

where the factor 100 comes from the gas-to-dust ratio (assumed to be constant and equal to the ISM value), $F_{890 \mu \mathrm{m}}$ is the $890 \mu \mathrm{m}$ flux, and $d$ is the distance (typically around $160 \mathrm{pc}$, and taken from the Gaia DR2 results for the individual objects in the sample). The factor $\kappa_{v}$ is the grain opacity (assumed to be $10 \mathrm{~cm}^{2} \mathrm{~g}^{-1}$ at $1000 \mathrm{GHz}$ with a power-law index of $\beta=1$; Beckwith et al. 1990). The $T_{\text {dust }}$ used here is $20 \mathrm{~K}$.

The assumed initial abundances of carbon and oxygen are also potentially important, in the context of volatile carbon depletion found in protoplanetary disks (e.g., Bergin et al. 2013; Favre et al. 2013; McClure et al. 2016). However, for CN this is less relevant. For all disk models discussed here, the initial overall abundances are the same as those used in the default models of Cazzoletti et al. (2018), since the CN abundance was found not to be sensitive to the depletion of volatile $\mathrm{C}$ and $\mathrm{O}$.

A final important assumption is the strength of the UV radiation field. As Cazzoletti et al. (2018) discuss, CN is very sensitive to the local abundance of excited molecular hydrogen $\mathrm{H}_{2}^{*}$, which in turn is sensitive to FUV line pumping. However, typically, no UV spectra exist for these sources (excepting RU Lup and RY Lup; Ardila et al. 2013; France et al. 2014; Arulanantham et al. 2018). We use a range of UV luminosities, where we focus on the $91.2-110 \mathrm{~nm}$ region. In that wavelength range, the UV luminosities sampled are between $1.5 \times 10^{-4}$ and $1.5 \times 10^{-6} L_{\odot}$ for models with a UV excess, versus $2.3 \times 10^{-11} L_{\odot}$ for models with a purely stellar radiation field. Using a rough approximation, this UV field can be linked to the accretion rate onto the star. Following Kama et al. (2016), the UV is produced from the accretion rate, the stellar mass, and a $10000 \mathrm{~K}$ black-body spectrum for the accreting material. Using this formulation an accretion rate of 

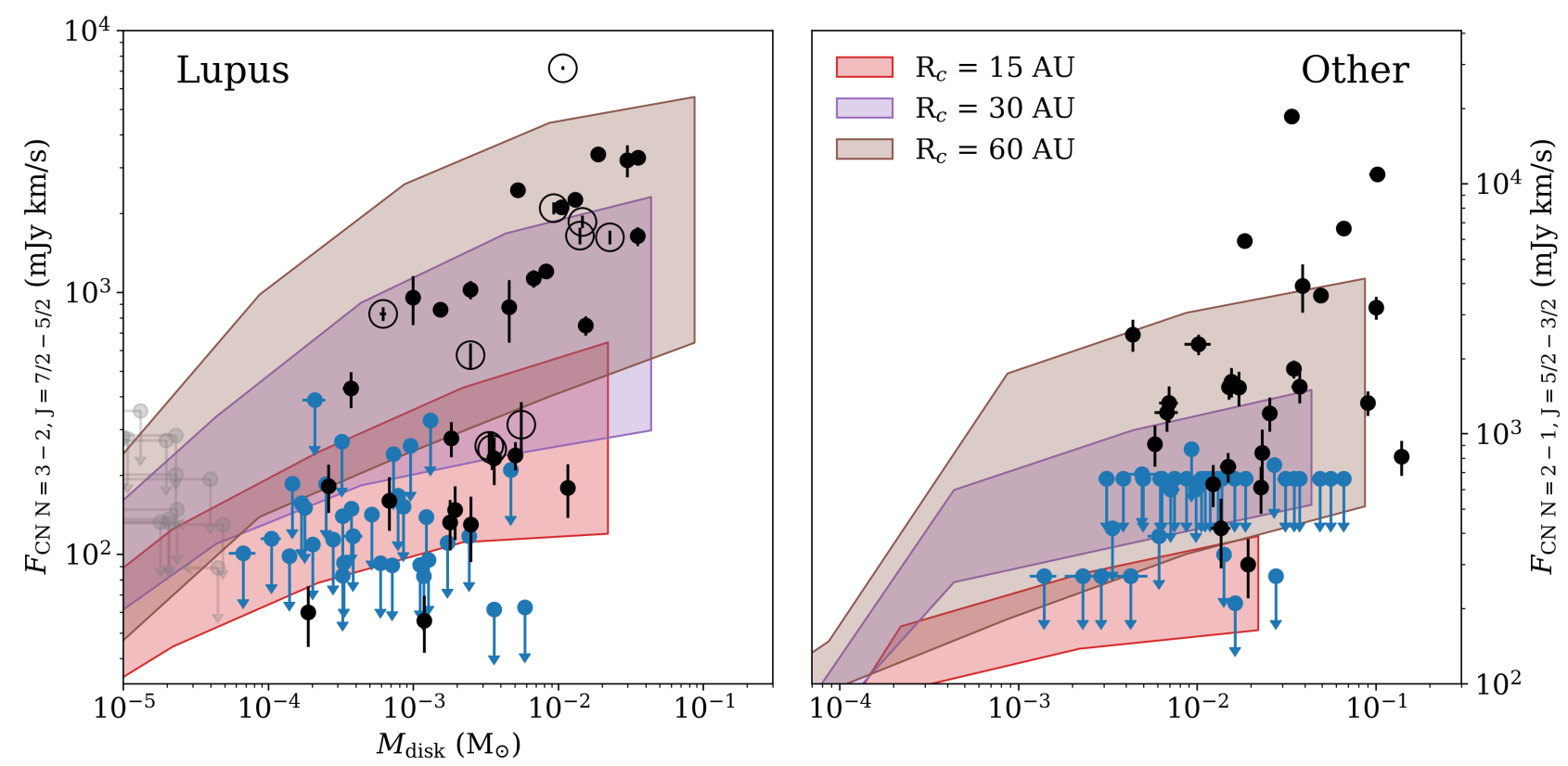

Fig. 3. Disk-integrated CN flux vs. disk mass for the Lupus disk sample (left panel) and the previously observed disks in Taurus-Auriga and $\rho$ Oph (Guilloteau et al. 2013; Reboussin et al. 2015; right panel). Detected sources are black, CN flux upper limits are blue, and double upper limits are gray. Colored regions indicate model results for the areas of parameter space covered by different UV fluxes and disk masses for a given $R_{\mathrm{c}}$. Transition disks are marked with a circle and were not modeled. Fluxes for the Taurus-Auriga and $\rho$ Oph sources and models are scaled to a $140 \mathrm{pc}$ distance.

$10^{-8} M_{\odot} \mathrm{yr}^{-1}$ corresponds to a UV luminosity of $1.5 \times 10^{-6} L_{\odot}$ but as will be shown below, this may be uncertain by as much as an order of magnitude.

\subsubsection{Linking $\mathrm{CN}$ to disk radii}

In Fig. 3, the model grid is overplotted to both the Lupus data and the previous large samples presented in Guilloteau et al. (2013) and Reboussin et al. (2015), after raytracing the appropriate transitions. The left panel shows the Lupus data and the corresponding $\mathrm{CN} N=3-2$ transition from the models; on the right panel the $N=2-1$ transition fluxes are shown. In this comparison, no assumptions on the line ratios have to be made, since we directly compare to the model fluxes. The colored areas show models with different UV luminosities and disk masses at given $R_{\mathrm{c}}$. From this figure, several conclusions can be drawn.

First, the large and unbiased disk population sampled in Lupus makes it apparent that for many of these disks the critical radius $R_{\mathrm{c}}$ must be small, even less than $15 \mathrm{AU}$, in order to reproduce the observed low $\mathrm{CN}$ fluxes. This result holds even when considering the impact of large variations in UV luminosity, and when considering variations in disk structure, as in Cazzoletti et al. (2018): the faintest detections and nondetections require compact gas disks. In the DALI models, the physical cause behind this strong effect is that, as the disk becomes more compact, the radius at which the $\mathrm{CN}$ emission would otherwise peak is located further out than the bulk of the disk material: the disk runs out before the $\mathrm{CN}$ can form in significant quantities.

Meanwhile, in line with Guilloteau \& Dutrey (1998) and the model results in Cazzoletti et al. (2018), the brightest disks in $\mathrm{CN}$ clearly require large radii, and values of $R_{\mathrm{c}}$ larger than $60 \mathrm{AU}$, suggesting that the observed Taurus sample contains more massive, but also more radially extended sources than are found, on average, in Lupus.
The observational determination of the $R_{\mathrm{c}}$ of a disk from resolved images is challenging, both for line- and continuum data. Even at high $\mathrm{S} / \mathrm{N}$, emission below the noise level may be missed. Moreover, gas- and continuum-derived outer radii for the same disk can differ: if a gas line is optically thick it may be detected further out than the dust (by a factor up to 3; Facchini et al. 2017). The inward drift and growth of dust grains further change the appearance of the continuum, while leaving the gas unaffected. Both of these effects have been shown in the Lupus star forming region (Ansdell et al. 2018, Trapman et al., in prep.). While a population of unresolved or marginally resolved dust disks had previously been identified in the uniformly deep continuum survey, our $\mathrm{CN}$ models allow us to conclude that their underlying density structure also has a compact gas surface density distribution when compared to well-studied disks like IM Lup (which has an $R_{\mathrm{c}}$ of $\left.60 \mathrm{AU}\right)$ or TW Hya $\left(R_{\mathrm{c}}=35 \mathrm{AU}\right.$; Cleeves et al. 2016; Huang et al. 2018).

\subsection{2. $\mathrm{CN}$ as a UV tracer}

The models run in Cazzoletti et al. (2018) and the grid used here both predict stronger $\mathrm{CN}$ emission from disks around stars that are more luminous in the $\mathrm{UV}$, due to the sensitivity of the $\mathrm{CN}$ chemical network to the abundance of $\mathrm{H}_{2}^{*}$. With the (known) stellar properties and accretion rates for the disk-bearing stars in Lupus, and the approximation for the UV excess introduced above for the DALI models, we can calculate a $91.2-110 \mathrm{~nm}$ UV flux for each object in the sample. In Fig. 4, these UV fluxes are compared to the observed $\mathrm{CN}$ flux. It is clear that, in contrast to the model predictions, no correlation exists between the $\mathrm{CN}$ flux and the UV flux estimated from the stellar flux and accretion rate, and this is confirmed by using a modified Kendall $\tau$-test.

This apparently contradictory result, however, can be reconciled with the model predictions. The accretion rates used to infer the UV flux of these disks are accurate up to a factor of a 


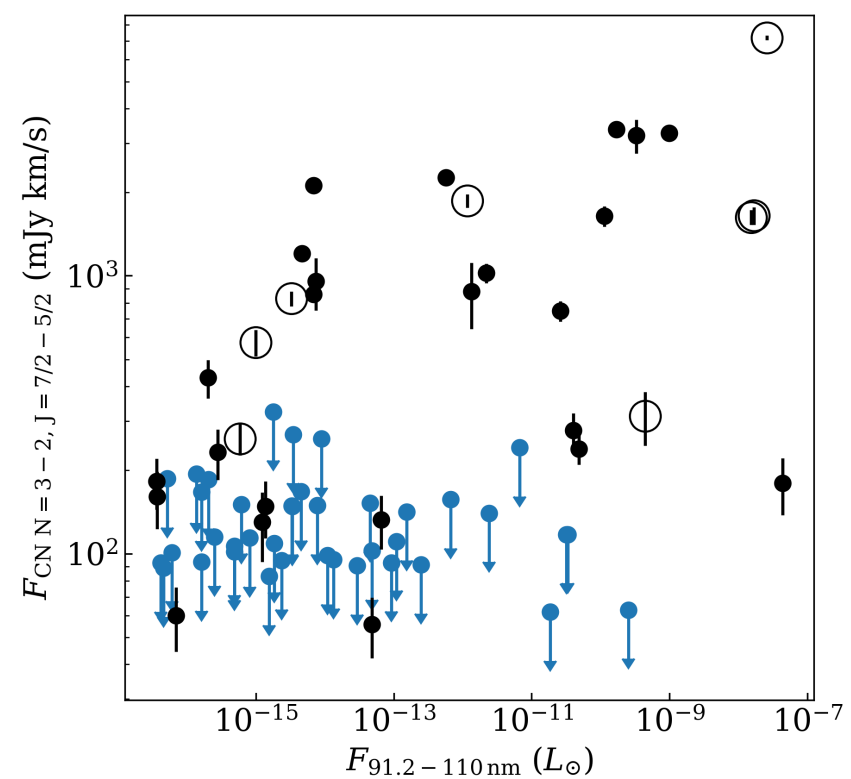

Fig. 4. Disk-integrated CN fluxes vs. inferred UV fluxes between 91.2 and $110 \mathrm{~nm}$ from the combined stellar luminosity and the accretion rate onto the star (Alcalá et al. 2017). Circles indicate transition disks.

few, being based on the Balmer continuum excess between 320 and $346 \mathrm{~nm}$ and optical line emission (Alcalá et al. 2017). More importantly, while the total excess UV should be proportional to the accretion rate $\dot{M}_{\text {acc }}$, it is not only emitted as continuum but also as line emission, which is more difficult to constrain (e.g., Herczeg et al. 2004; Bergin et al. 2004). In particular, the lines between 91.2 and $110 \mathrm{~nm}$ are relevant, since they may overlap with UV pumping lines for $\mathrm{H}_{2}$ to create $\mathrm{H}_{2}^{*}$ via UV pumping. From previous studies of the UV lines of protoplanetary disks at these wavelengths, strong emission lines in this wavelength range have been shown to be present, and responsible for a significant fraction of UV radiation (e.g., Herczeg et al. 2005; Ardila et al. 2013; France et al. 2014).

One way to provide extra, independent information on the UV excess is to use the spatially resolved data, as opposed to the disk-integrated fluxes discussed here. This is because, as demonstrated in Fig. 11 of Cazzoletti et al. (2018), the radial intensity profile of $\mathrm{CN}$ is also very sensitive to the UV radiation field. In the following, two disks for which $\mathrm{CN}$ is spatially resolved are discussed in greater detail.

\section{4. $\mathrm{CN}$ images}

\subsection{Observations of $S z 98$ and $S z 71$}

The targets selected for detailed discussion in this section, $\mathrm{Sz} 98$ and Sz71, are the second and sixth brightest object in $\mathrm{CN}$, respectively. These disks are particularly suited to modeling: their data have high $\mathrm{S} / \mathrm{N}$ and show clear evidence of a ring-like emission morphology, as well as well-resolved emission. Based on their continuum emission, both disks have a favorable, relatively face-on inclination $\left(45^{\circ}\right.$ for $\mathrm{Sz} 98,36.5^{\circ}$ for $\left.\mathrm{Sz} 71\right)$. The stars do not cover the peak of the spectral type distribution in Lupus, which lies between M3 and M4: Sz 71 is an M1.5 star and $\mathrm{Sz} 98$ is a $\mathrm{K} 7$ star. The stellar properties used for the sources in this section are listed in Table 1.

Figure 5 presents the moment-zero maps as well as the disk continuum, showing $\mathrm{CN}$ and the bulk-gas tracers ${ }^{13} \mathrm{CO} J=3-2$ and ${ }^{12} \mathrm{CO} J=2-1$. In Fig. 6 , the radial intensity profiles of the continuum and $\mathrm{CN}$ line for both sources are shown. Maps of the individual channels (at $0.5 \mathrm{~km} \mathrm{~s}^{-1}$ resolution) are shown in Figs. D.1 and D.2. Both sources show $\mathrm{CN}$ rings, while Sz 98 shows evidence of significant but noncoincident continuum substructure. To improve the sensitivity of our analysis, these profiles have been made by azimuthally averaging over the (deprojected) moment-zero maps. In Fig. D. 3 the spectra of these lines are shown; channel maps of the $\mathrm{CN}$ emission towards both sources can be found in Appendix D. At the $3 \sigma$-level, $\mathrm{C}^{18} \mathrm{O}$ is not detected towards either source. Table 2 shows the integrated fluxes for the lines discussed here, as well as the source position angles (PAs) and inclinations derived with the imfit task in CASA.

The other CN-bright sources are less suited for this purpose. A large fraction of the sources are too compact for structure in the $\mathrm{CN}$ emission to be apparent; $28 \%$ of the $\mathrm{CN}$-detected sources are unresolved. Of the rest, many have low S/N levels, which make their detailed image-plane analysis impossible. Some of the brightest disks in $\mathrm{CN}$ are transition disks, which present a unique continuum structure with a large central cavity and are exposed to a different UV radiation field as a consequence. This may change the typical behavior of CN. Of the full disks, V1094 Sco is both radially extended and bright, both in continuum and gas emission, but its $\mathrm{CN}$ line is partly resolved-out at the largest scales in low-velocity channels, and optically thick continuum emission in the inner disk may obscure the $\mathrm{CN}$ emission in those parts (van Terwisga et al. 2018). For IM Lup, only Submillimeter Array (SMA) data for $\mathrm{CN}$ are available and at much lower resolution, and $\mathrm{Sz} 133$ is edge-on.

\subsubsection{CN 3-2}

In both disks, CN 3-2 emission is ring-like, despite the relatively high noise levels of the final moment-zero maps. The peak emission is found at 40 and $60 \mathrm{AU}$ for $\mathrm{Sz} 71$ and Sz 98, respectively, although the emission does not completely vanish in the central region of the disk. The $\mathrm{CN}$ emission extends to large radii in both sources, and is clearly more extended than the continuum: in $\mathrm{Sz} 71, \mathrm{CN}$ is detected out to approximately $200 \mathrm{AU}$, and in $\mathrm{Sz} 98$ it can be seen out to $250 \mathrm{AU}$, while the continuum outer radii for both disks are 150 and $200 \mathrm{AU}$, respectively. The peak emission radius is further from the star in Sz 98 than in $\mathrm{Sz} 71$. The latter source has the smallest contrast between emission at the disk center and peak intensity of these two sources.

In the moment-zero maps of $\mathrm{CN}$, some asymmetry in the emission can be observed: this is likely due to the second-brightest hyperfine structure component $(J=7 / 2-5 / 2$, $F=5 / 2-3 / 2$ ) at $0.5 \mathrm{~km} \mathrm{~s}^{-1}$ from the targeted transition which is blended with the strongest hyperfine transition lines of $F=$ $9 / 2-7 / 2$ and $F=7 / 2-5 / 2$, contaminating the emission redward of the primary hyperfine line. The theoretical relative intensity is expected to be up to $30 \%$ of the primary (Kastner et al. 2014; Hily-Blant et al. 2017). However, this does not appear to significantly impact the radial profile observed for the source, apart from some increase in noise in the most affected areas.

Another possible influence on the $\mathrm{CN}$ morphology is the existence of optically thick continuum, blanketing the lines. However, we can exclude this possibility: the observed brightness temperatures at the source center (where such an effect would be the most likely) of 6.0 and $4.4 \mathrm{~K}$ for $\mathrm{Sz} 71$ and $\mathrm{Sz} 98$, respectively, are too low to be consistent with an optically thick continuum if an effective dust temperature of $20 \mathrm{~K}$ is assumed. This midplane temperature value is not unlikely given the relatively large beam size of our observations. Moreover, 

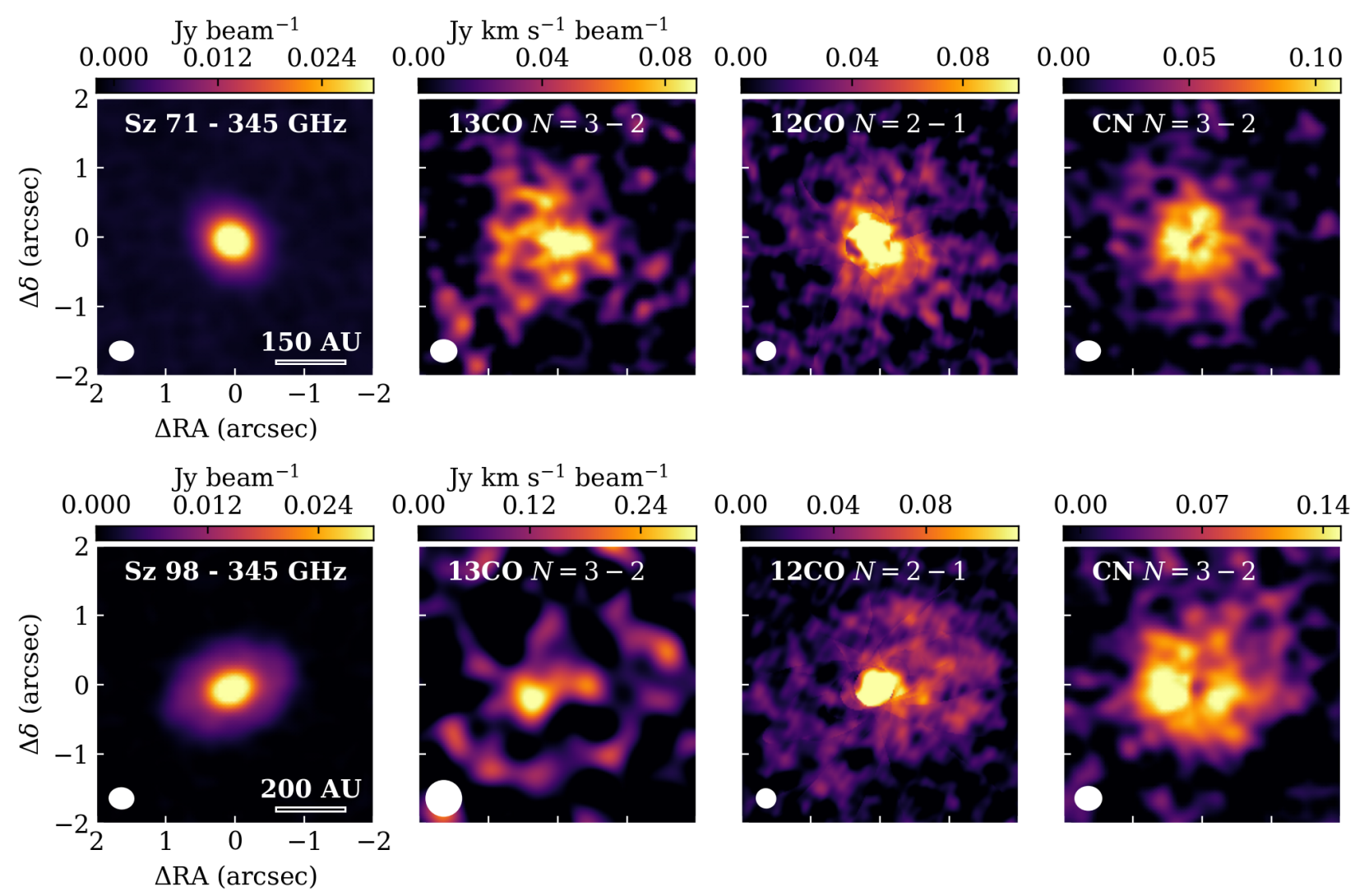

Fig. 5. Continuum and moment-zero maps of ${ }^{13} \mathrm{CO} 3-2,{ }^{12} \mathrm{CO} 2-1$, and $\mathrm{CN} N=3-2$ (including only the brightest $J=7 / 2-5 / 2$ transitions) for both sources.
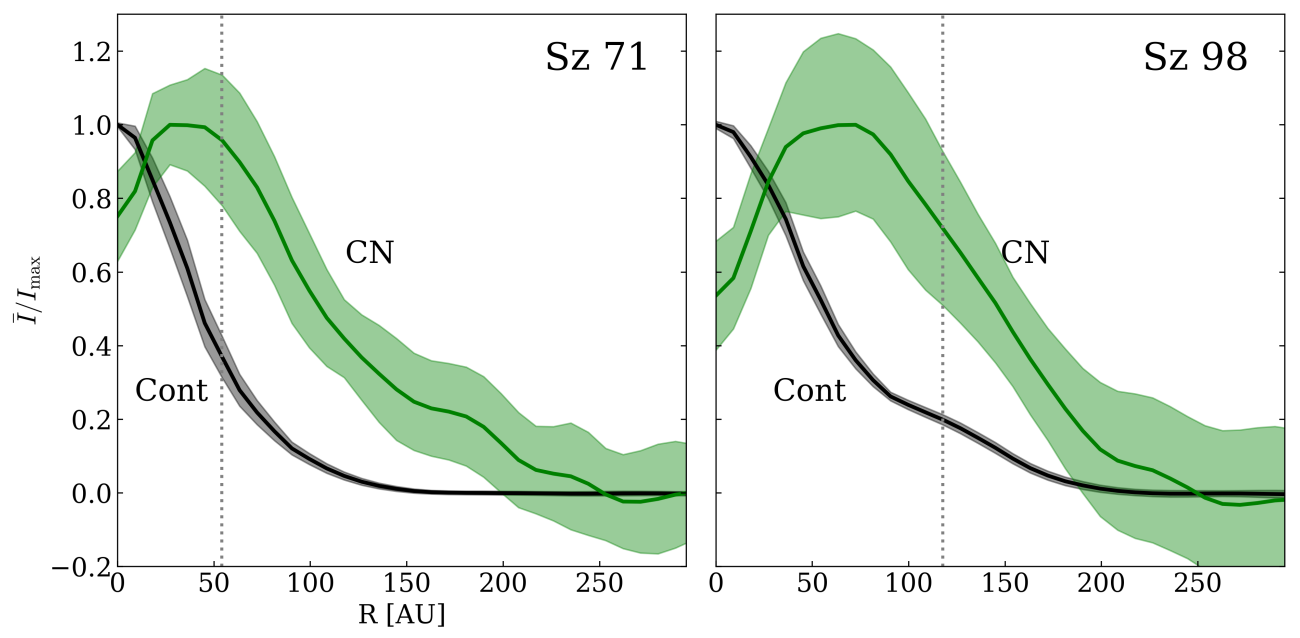

Fig. 6. Radial cuts of the continuum (black) and $\mathrm{CN}$ moment-zero (green) maps for both sources, normalised to the peak radially averaged intensity, after deprojecting and derotating the imageplane data. The shaded regions indicate the $1 \sigma$ noise level. The gray dotted line indicates the location of a local maximum in the continuum due to an (unresolved) dust ring in $\mathrm{Sz}$ 98, and the location of continuum knee in $\mathrm{Sz} 71$ (see Appendix B). we do detect $\mathrm{CO}$ inside the $\mathrm{CN}$ rings in both sources, and unambiguously detect ${ }^{13} \mathrm{CO}$ in the $\mathrm{CN}$ hole location in $\mathrm{Sz} 71$.

\subsection{2. ${ }^{12} \mathrm{CO} 2-1$}

${ }^{12} \mathrm{CO}$ is a bright and optically thick line in the atmospheres of protoplanetary disks, but it is strongly affected by absorption by foreground clouds towards both sources discussed here, at several separate velocity ranges. By inspecting the spectra in Fig. D.3, some cloud absorption appears to be present in the ${ }^{12} \mathrm{CO}$ line. This is consistent with single-dish observations of CO in the Lupus clouds (van Kempen et al. 2007). Cloud absorption should not have a similar effect on our $\mathrm{CN}$ data: the $N=3-2$ transition has a much higher critical density (of $n_{\text {crit }} \sim 6.0 \times 10^{6} \mathrm{~cm}^{-3}$ ) than the low-density foreground cloud, and no evidence of cloud contamination of $\mathrm{CN}$ is found by Guilloteau et al. (2013) in Taurus.

Despite the absorption, there is sufficient signal to infer an outer radius for the ${ }^{12} \mathrm{CO}$ emission: the low-velocity channels blueward of the source velocity are not very absorbed. The outer radius at which $\mathrm{CO}$ is detected is larger than that of the $\mathrm{CN}$ emission radius towards both sources. Both $\mathrm{Sz} 98$ and $\mathrm{Sz} 71$ have detectable ${ }^{12} \mathrm{CO}$ out to about $250 \mathrm{AU}$, where the outer radius is limited by the $\mathrm{S} / \mathrm{N}$ of our observations. Thus the gas extends at least a factor of $\sim 2-3$ further than the continuum in both disks, which is typical for most Lupus disks (Ansdell et al. 2018). 
Table 1. Stellar properties of $\mathrm{Sz} 98$ and $\mathrm{Sz} 71$.

\begin{tabular}{|c|c|c|c|c|c|}
\hline & $\mathrm{SpT}$ & $\begin{array}{l}d \\
(\mathrm{pc}) \\
(1)\end{array}$ & $\begin{array}{l}L_{\star} \\
\left(L_{\odot}\right) \\
(2)\end{array}$ & $\begin{array}{l}M_{\star} \\
\left(M_{\odot}\right) \\
(2)\end{array}$ & $\begin{array}{l}\log \left(\dot{M}_{\mathrm{acc}}\right) \\
M_{\odot} \mathrm{yr}^{-1} \\
(2)\end{array}$ \\
\hline Sz 71 & M1.5 & 155.89 & 0.33 & 0.42 & -9.03 \\
\hline $\mathrm{Sz} 98$ & $\mathrm{~K} 7$ & 156.22 & 1.53 & 0.74 & -7.59 \\
\hline
\end{tabular}

References. (1) Comerón (2008); (2) Alcalá et al. (2017).

\subsection{3. ${ }^{13} \mathrm{CO} 3-2$}

The ${ }^{13} \mathrm{CO}$ emission towards both sources is surprisingly faint and thus the maps suffer from low S/N (Ansdell et al. 2016; Miotello et al. 2017). Towards Sz 98, the detection of the line is marginal. Binning the data to $1 \mathrm{~km} \mathrm{~s}^{-1}$-wide channels and lowering the resolution to $0.6^{\prime \prime}$ by removing short baselines improves our sensitivity. The moment-zero map of ${ }^{13} \mathrm{CO}$ in this source does not show emission across the full disk, but only in "lobes" towards the sides; this is probably caused by the low $\mathrm{S} / \mathrm{N}$ of the data, as well as the limb-brightening effect of optically thin emission. In $\mathrm{Sz} 71$, the emission is brighter and clearly centrally peaked, but also faint. Because of the low surface brightness of the line and the large $\mathrm{S} / \mathrm{N}$ difference between the sources, we have not included ${ }^{13} \mathrm{CO}$ radial cuts in Fig. 6.

The faintness of ${ }^{13} \mathrm{CO}$ in $\mathrm{Sz} 98$ is especially surprising: it is one of the largest and brightest (in both ${ }^{12} \mathrm{CO}$ and continuum) disks in the Lupus sample, even including transition disks. Comparing the regions of the spectrum where ${ }^{12} \mathrm{CO}$ is absorbed to the ${ }^{13} \mathrm{CO}$ spectrum in $\mathrm{Sz} 71$ (Fig. D.3, top) shows that foreground absorption is unlikely to be the full explanation for the faintness of the ${ }^{13} \mathrm{CO}$ line.

\subsubsection{CN versus continuum}

It is possible that, similar to the situation for $\mathrm{CO}$ (e.g., Isella et al. 2016; Fedele et al. 2017), a link exists between continuum structure and the radial distribution of $\mathrm{CN}$. Of the two disks discussed here, $\mathrm{Sz} 98$ has the most obvious continuum feature: as shown in Fig. 6, based on the $u v$-plane fit in Appendix B, a secondary maximum exists at the location of the "bump" in the continuum at $\sim 120 \mathrm{AU}$. No counterpart of this feature is seen in the $\mathrm{CN}$ emission profile: $\mathrm{CN}$ peaks at a radius interior to it, and seems to present a fairly constant downward slope outside $100 \mathrm{AU}$.

For $\mathrm{Sz} 71$, the continuum structure is less obvious: around $50 \mathrm{AU}$, the continuum profile begins to drop off less steeply. This is possibly related to an unresolved feature, but this is not clear even from the $u v$-plane. The dashed line in Fig. 6 for this object indicates the point where the turnover to a less steep profile occurs; it is fairly close to the $\mathrm{CN}$ peak.

Given the differences in the comparison between continuum and $\mathrm{CN}$ for these two sources, we therefore conclude that no obvious relation between the two is present in all cases. The clear presence of ring-like $\mathrm{CN}$ emission profiles in $\mathrm{Sz} 71$ and $\mathrm{Sz} 98$ is consistent with the predictions for rings of $\mathrm{CN}$ emission (Cazzoletti et al. 2018), and suggests that these features may be the result of chemistry alone, without the need for underlying continuum structures.

\subsection{DALI modeling of $\mathrm{CN}$ rings}

Here, a purely chemical cause for $\mathrm{CN}$ rings is investigated by more detailed modeling of the two bright, resolved disks discussed previously. The thermo-chemical disk modeling code DALI was used to test if simple models without the dust substructures identified in the previous subsection can produce radial distributions and integrated intensities of $\mathrm{CN}$ similar to those observed.

Based on the results of Cazzoletti et al. (2018), the presence of $\mathrm{CN}$ rings appears to be a common feature for their grid of protoplanetary disk models. Our goal is not to provide a perfect fit to the data, but rather to investigate whether rings similar (in terms of total flux and radial profile) to those observed in these two disks can be produced without continuum substructure, and to isolate the main parameters governing $\mathrm{CN}$ emission strength and morphology. It is important in this context to note that in an absolute sense the uncertainties resulting from the assumptions in the chemical network alone, as well as from disk structure, lead to an uncertainty of a factor of a few in flux (Woitke et al. 2018).

\subsubsection{Individual disk model details}

In order to find a rough physical structure of the disk models, we first attempted to reproduce the objects' spectral energy distribution (SED), described in more detail in Appendix C. This ensures that the large-scale distribution of the dust temperatures in the DALI models is similar to that of the source, and allows us to find, for instance, the $\mathrm{CO}$ snowline in the disks. Again, a parametric dust distribution model, as in Andrews et al. (2011), was used, constrained by data spanning a wide range of wavelengths between visible and mm-wavelengths. The gas surface density is $100 \times$ the dust surface density and is not decoupled. The disk structure parameters used for the models of both disks have not been varied further in their respective DALI models and can be found in Table C.1. The outer radii of the disks were taken to be $200 \mathrm{AU}$, based on the $\mathrm{CN}$ data. The stellar parameters adopted for the input photosphere in the models were taken from Alcalá et al. (2014, 2017). As before, an initial estimate of the relevant UV flux for CN formation, $L_{912-1100 \AA}$, the UV excess emission from the model star was parametrized by the mass accretion rate, assuming $10000 \mathrm{~K}$ black-body emission from the accreted material; due to the aforementioned uncertainties we have allowed for variations in the UV excess at these wavelengths of up to a factor 10 .

A model of $\mathrm{Sz} 71$ with a UV excess of $\log \left(L_{912-1100 \AA} / L_{\odot}\right)=-5.2$, based on the measured accretion rate, as well as a model with an increased UV excess luminosity of $\log \left(L_{912-1100 \AA} / L_{\odot}\right)=-4.1$, denoted as M1 and M2, respectively, are presented in this section. For $\mathrm{Sz} 98$, we only ran models with a single UV luminosity based on the observed accretion properties, and thus only have an M1, since this disk model already performed quite well in reproducing the $\mathrm{CN}$ flux and radial profile. The surface density profiles were kept fixed for all models of a single source. After ray-tracing each model, the images were convolved with a $0.3^{\prime \prime}$ circular Gaussian beam, to see if the $\mathrm{CN}$ emission was similar to the observations.

\subsubsection{Model results}

As can be seen in Fig. 7, the most remarkable feature of the $\mathrm{CN}$ emission, its concentration in a ring, is indeed reproduced in the model radial profiles, and occurs at radii within 20 AU of those seen in the data for $\mathrm{Sz} 71$ model M2 and Sz 98 model M1. Also, the peak intensity of the emission is close (within 40\%) to the observed peak value for the most similar models. The similarity in the emission profiles outside the peak is also encouraging, 

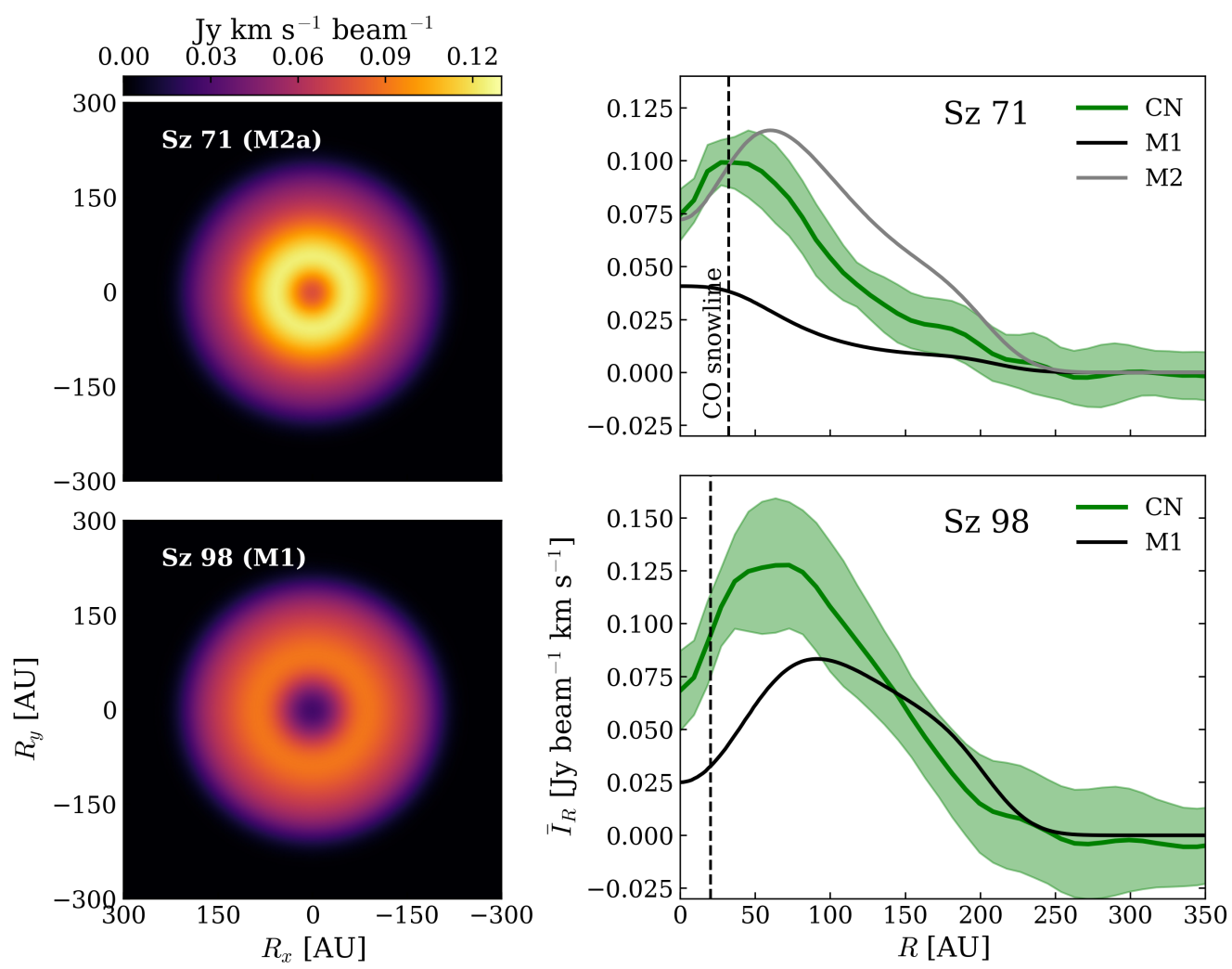

Fig. 7. CN model images and radial intensity profiles (black and gray) from the DALI models, after convolving with a $0 .{ }^{\prime \prime} 3$ beam for both sources. Model M2 for Sz 71 has an increased UV luminosity relative to M1. The black dashed vertical lines indicate (approximately) the CO snow line locations in the models. The observed radial profiles of $\mathrm{CN}$ are shown in green, together with their $1 \sigma$ errors.

Table 2. Disk emission properties for $\mathrm{Sz} 71$ and $\mathrm{Sz} 98$.

\begin{tabular}{lllllll}
\hline \hline & $\begin{array}{l}F_{345 \mathrm{GHz}} \\
(\mathrm{mJy})\end{array}$ & $\begin{array}{l}F_{12} \mathrm{CO} \mathrm{2-1}^{2} \\
\left(\mathrm{Jy} \mathrm{km} \mathrm{s}^{-1}\right)\end{array}$ & $\begin{array}{l}F_{13} \mathrm{CO} \mathrm{3-2} \\
\left(\mathrm{Jy} \mathrm{km} \mathrm{s}^{-1}\right)\end{array}$ & $\begin{array}{l}F_{\mathrm{CN} \mathrm{3-2}} \\
\left(\mathrm{Jy} \mathrm{km} \mathrm{s}^{-1}\right)\end{array}$ & $\begin{array}{l}\mathrm{PA}^{a} \\
\left({ }^{\circ}\right)\end{array}$ & $\begin{array}{l}i^{a} \\
\left.{ }^{\circ}\right)\end{array}$ \\
\hline Sz 71 & $166.04 \pm 0.63$ & $2.86 \pm 0.26^{b}$ & $1.3 \pm 0.1$ & $2.35 \pm 0.95$ & -37.5 & 47.0 \\
Sz 98 & $237.29 \pm 1.42$ & $2.99 \pm 0.22^{b}$ & $0.51 \pm 0.1$ & $3.49 \pm 0.13$ & 107.4 & -40.8 \\
\hline
\end{tabular}

Notes. ${ }^{(a)}$ Derived from the continuum data using the CASA imfit-task. ${ }^{(b)}$ Affected by foreground absorption and thus a lower limit.

Table 3. Parameter values and integrated CN fluxes for the DALI models of $\mathrm{Sz} 71$ and $\mathrm{Sz} 98$.

\begin{tabular}{llll}
\hline \hline & & $\log \left(L_{912-1100 \AA} / L_{\odot}\right)$ & $\begin{array}{l}F_{\mathrm{CN}} \\
\left(\mathrm{Jy} \mathrm{km} \mathrm{s}^{-1}\right)\end{array}$ \\
\hline Sz 71 & M1 & -5.2 & 0.8 \\
& M2 & -4.1 & 3.9 \\
& Obs & & 2.4 \\
Sz 98 & M1 & -3.4 & 3.7 \\
& Obs & & 3.5 \\
\hline
\end{tabular}

Notes. The most representative model is indicated in boldface.

especially considering the uncertainty of a factor of a few in absolute fluxes from this type of chemical model.

Table 3 presents the total CN fluxes for the different models. The agreement is closest for Sz 98: the model flux and observed flux only differ by $5 \%$. For Sz 71, if an increased UV luminosity is used, a larger difference can be seen, of $\sim 60 \%$ for all models; but the emission profile for M2 is clearly closer than that of model M1 for this disk, primarily due to the increase in peak intensity radius with increased UV flux.

No clear link appears between the CO snow-line position (defined as the location where the disk midplane reaches $20 \mathrm{~K}$, following Öberg et al. 2005) and the location of the $\mathrm{CN}$ peak in $\mathrm{Sz} 98$. For Sz 71, the CO snow line, CN peak, and - possibly - the continuum slope change lie within $10 \mathrm{AU}$, but the location of the snow line is quite uncertain, and both it and the continuum structure may not be closely related to the $\mathrm{CN}$ emission, which generally arises from the upper parts of the disk in models (Cazzoletti et al. 2018). In either case, the significant difference between the radii of the expected $\mathrm{CO}$ snow line position, the CN peak, and the continuum structure in $\mathrm{Sz} 98$ seem to preclude a clear, one-to-one link between these quantities in all disks.

\subsection{3. $\mathrm{CN}$ rings as a disk probe}

The main factor determining the $\mathrm{CN}$ radius and emission strength in these large-disk models is the excess UV flux due 
to accretion of material onto the star. This is especially obvious from comparing models M1 and M2 for Sz 71. It is crucial to note that in none of our models do we need the presence of substructure in the disks' surface density, either in the dust or gas components: $\mathrm{CN}$ rings arise purely from a chemical effect.

In the wider grid of disks in Cazzoletti et al. (2018), as well as in these models, $\mathrm{CN}$ rings are identified as a common feature. However, the ring radii and fluxes decrease with stellar $T_{\text {eff }}$ and disk size, which explains why we can only study them in these two sources in the survey: below $R_{\mathrm{c}}=40 \mathrm{AU}$, which applies to most of the objects in our sample, the ring radius drops rapidly and starts to become unresolved at our observations' effective resolution. At the same time, the flux and therefore $\mathrm{S} / \mathrm{N}$ of the resolved $\mathrm{CN}$ images fall off. In the grid of models discussed in Sect. 3, the smallest $\mathrm{CN}$ peak radii would be resolved with an effective beam size of $\sim 10 \mathrm{AU}$, given a face-on inclination and sufficiently good $\mathrm{S} / \mathrm{N}$.

Ultimately, $\mathrm{CN}$ is best used as a probe of the $91.2-110 \mathrm{~nm}$ UV radiation field impacting the upper disk. This is especially the case if the other disk parameters, particularly the characteristic radius $R_{\mathrm{c}}$, can be constrained independently. For this, other common molecular tracers like ${ }^{12} \mathrm{CO}$ or ${ }^{13} \mathrm{CO}$ can be used, leaving the way open for such studies in the future.

\section{Conclusions}

Our ALMA observations of a 99\% complete sample of Class-II disks in Lupus allow us to study the behavior of $\mathrm{CN}$, both in the full sample and - using individual models - in two resolved sources, and to use this common molecule as a probe of upperdisk atmosphere properties. With higher resolution and deeper observations, this molecule can be used to gain essential information on the properties of protoplanetary disks. The main results obtained from the observations discussed here are as follows:

- The CN $N=3-2, J=7 / 2-5 / 2$ transition is bright, and has a similar detection rate to ${ }^{13} \mathrm{CO}$ in disks in Lupus, but is not strongly correlated with either ${ }^{13} \mathrm{CO}$ or continuum fluxes in these disks.

- Comparing the $\mathrm{CN}$ flux distribution in Lupus to that of a population of Taurus-Auriga disks shows no significant difference, while disks in $\rho$ Oph may be fainter on average.

- Comparing the CN fluxes of the full Lupus sample to a model grid with varying UV fluxes and gas disk characteristic radii, $R_{\mathrm{c}}$, supports the conclusion that a significant number of disks in Lupus has a compact gas surface density profile, with $R_{\mathrm{c}} \leq 15 \mathrm{AU}$ needed to explain part of the population.

- CN shows a ring-shaped emission morphology towards the bright, resolved sources $\mathrm{Sz} 71$ and $\mathrm{Sz} 98$, consistent with model predictions that $\mathrm{CN}$ is generally distributed in rings. For the bulk of objects, it is not possible to detect rings at this sensitivity and resolution, consistent with the expectations for compact, low-mass disks around late-type stars.

- Continuum substructure unrelated to the $\mathrm{CN}$ rings appears to be present in both sources, and particularly in $\mathrm{Sz} 98$, where we infer an unresolved continuum ring at $\sim 120$ AU. No connection between the $\mathrm{CO}$ snow line, the continuum structure, and the $\mathrm{CN}$ ring are seen in $\mathrm{Sz} 98$.

- Disk models without substructure retrieve CN rings similar to those observed towards both sources, in terms of both peak emission radii and absolute intensities. Their radii are strongly dependent on the amount of excess UV emission, implying that $\mathrm{CN}$ rings are purely chemical in nature, and good tracers of the effective UV irradiation of the upper disk atmosphere, especially if gas disk sizes are determined from other data.

Acknowledgements. We kindly thank J. M. Alcalá for providing us with X-shooter spectra of both sources, and A. Bosman, and D. Harsono for useful discussions. We also thank S. Bruderer for his role in the continued development of DALI and his advice on how best to use it. Astrochemistry in Leiden is supported by the European Union A-ERC grant 291141 CHEMPLAN, by the Netherlands Research School for Astronomy (NOVA), and by a Royal Netherlands Academy of Arts and Sciences (KNAW) professor prize. This paper makes use of the following ALMA data: ADS/JAO.ALMA\#2013.1.00220.S and ADS/JAO.ALMA\#2015.1.00222.S. ALMA is a partnership of ESO (representing its member states) NSF (USA) and NINS (Japan) together with NRC (Canada) MOST and ASIAA (Taiwan), and KASI (Republic of Korea), in cooperation with the Republic of Chile. The Joint ALMA Observatory is operated by ESO, AUI/NRAO and NAOJ. The authors acknowledge support by Allegro, the European ALMA Regional Center node in The Netherlands, and expert advice from L.T. Maud in particular. M.T. has been supported by the DISCSIM project, grant agreement 341137 funded by the European Research Council under ERC-2013-ADG. S.F., C.F.M. and A.M. acknowledge an ESO Fellowship.

\section{References}

Aikawa, Y., Furuya, K., Nomura, H., \& Qi, C. 2015, ApJ, 807, 120 Alcalá, J. M., Natta, A., Manara, C. F., et al. 2014, A\&A, 561, A2 Alcalá, J. M., Manara, C. F., Natta, A., et al. 2017, A\&A, 600, A20 ALMA Partnership (Brogan, C. L., et al.) 2015, ApJ, 808, L3 Andrews, S. M., \& Williams, J. P. 2005, ApJ, 631, 1134

Andrews, S. M., Wilner, D. J., Espaillat, C., et al. 2011, ApJ, 732, 42 Andrews, S. M., Wilner, D. J., Zhu, Z., et al. 2016, ApJ, 820, L40 Ansdell, M., Williams, J. P., van der Marel, N., et al. 2016, ApJ, 828, 46 Ansdell, M., Williams, J. P., Trapman, L., et al. 2018, ApJ, 859, 21 Ardila, D. R., Herczeg, G. J., Gregory, S. G., et al. 2013, ApJS, 207, 1 Arulanantham, N., France, K., Hoadley, K., et al. 2018, ApJ, 855, 98 Bailer-Jones, C. A. L., Rybizki, J., Fouesneau, M., Mantelet, G., \& Andrae, R. 2018, AJ, 156, 58

Barenfeld, S. A., Carpenter, J. M., Ricci, L., \& Isella, A. 2016, ApJ, 827, 142 Beckwith, S. V. W., Sargent, A. I., Chini, R. S., \& Guesten, R. 1990, AJ, 99, 924

Bergin, E., Calvet, N., Sitko, M. L., et al. 2004, ApJ, 614, L133

Bergin, E. A., Cleeves, L. I., Gorti, U., et al. 2013, Nature, 493, 644

Bergin, E. A., Du, F., Cleeves, L. I., et al. 2016, ApJ, 831, 101

Bruderer, S. 2013, A\&A, 559, A46

Bruderer, S., van Dishoeck, E. F., Doty, S. D., \& Herczeg, G. J. 2012, A\&A, 541, A91

Bustamante, I., Merín, B., Ribas, Á., et al. 2015, A\&A, 578, A23

Carney, M. T., Hogerheijde, M. R., Loomis, R. A., et al. 2017, A\&A, 605, A21

Cazzoletti, P., van Dishoeck, E. F., Visser, R., Facchini, S., \& Bruderer, S. 2018 A\&A, 609, A93

Cieza, L. A., Ruíz-Rodríguez, D., Hales, A., et al. 2019, MNRAS, 482, 698

Cleeves, L. I., Öberg, K. I., Wilner, D. J., et al. 2016, ApJ, 832, 110

Comerón, F. 2008, in Handbook of Star Forming Regions, Volume II: The Southern Sky, ed. B. Reipurth (San Francisco: ASP Monograph Publications), 5 295

Cutri, R. M., Skrutskie, M. F., van Dyk, S., et al. 2003, VizieR Online Data Catalog: II $/ 246$

Dullemond, C. P., \& Dominik, C. 2005, A\&A, 434, 971

Dutrey, A., Guilloteau, S., \& Guelin, M. 1997, A\&A, 317, L55

Espaillat, C., Muzerolle, J., Najita, J., et al. 2014, Protostars and Planets VI (Tucson: University of Arizona Press), 497

Facchini, S., Birnstiel, T., Bruderer, S., \& van Dishoeck, E. F. 2017, A\&A, 605, A16

Favre, C., Cleeves, L. I., Bergin, E. A., Qi, C., \& Blake, G. A. 2013, ApJ, 776, L38

Fedele, D., Carney, M., Hogerheijde, M. R., et al. 2017, A\&A, 600, A72

France, K., Schindhelm, E., Bergin, E. A., Roueff, E., \& Abgrall, H. 2014, ApJ, 784, 127

Gaia Collaboration (Brown, A. G. A., et al.) 2018, A\&A, 616, A1

Guilloteau, S., \& Dutrey, A. 1998, A\&A, 339, 467

Guilloteau, S., Di Folco, E., Dutrey, A., et al. 2013, A\&A, 549, A92

Guilloteau, S., Simon, M., Piétu, V., et al. 2014, A\&A, 567, A117

Guilloteau, S., Reboussin, L., Dutrey, A., et al. 2016, A\&A, 592, A124

Heays, A. N., Bosman, A. D., \& van Dishoeck, E. F. 2017, A\&A, 602, A105

Henning, T., \& Semenov, D. 2013, Chem. Rev., 113, 9016 
Herczeg, G. J., Wood, B. E., Linsky, J. L., Valenti, J. A., \& Johns-Krull, C. M. 2004, ApJ, 607, 369

Herczeg, G. J., Walter, F. M., Linsky, J. L., et al. 2005, AJ, 129, 2777

Hily-Blant, P., Magalhaes, V., Kastner, J., et al. 2017, A\&A, 603, L6

Huang, J., Öberg, K. I., Qi, C., et al. 2017, ApJ, 835, 231

Huang, J., Andrews, S. M., Cleeves, L. I., et al. 2018, ApJ, 852, 122

Isella, A., Guidi, G., Testi, L., et al. 2016, Phys. Rev. Lett., 117, 25110

Isobe, T., Feigelson, E. D., \& Nelson, P. I. 1986, ApJ, 306, 490

Kama, M., Bruderer, S., van Dishoeck, E. F., et al. 2016, A\&A, 592, A83

Kastner, J. H., Hily-Blant, P., Rodriguez, D. R., Punzi, K., \& Forveille, T. 2014, ApJ, 793, 55

Kastner, J. H., Qi, C., Gorti, U., et al. 2015, ApJ, 806, 75

Lebouteiller, V., Barry, D. J., Goes, C., et al. 2015, ApJS, 218, 21

Lique, F., Spielfiedel, A., Feautrier, N., et al. 2010, J. Chem. Phys., 132, 024303

Loison, J.-C., Wakelam, V., \& Hickson, K. M. 2014, MNRAS, 443, 398

Long, F., Herczeg, G. J., Pascucci, I., et al. 2017, ApJ, 844, 99

Loomis, R. A., Öberg, K. I., Andrews, S. M., et al. 2018, AJ, 155, 182

Lynden-Bell, D., \& Pringle, J. E. 1974, MNRAS, 168, 603

Mathews, G. S., Klaassen, P. D., Juhász, A., et al. 2013, A\&A, 557, A132

McClure, M. K., Bergin, E. A., Cleeves, L. I., et al. 2016, ApJ, 831, 167

Miotello, A., van Dishoeck, E. F., Williams, J. P., et al. 2017, A\&A, 599, A113

Öberg, K. I., van Broekhuizen, F., Fraser, H. J., et al. 2005, ApJ, 621, L33

Öberg, K. I., Qi, C., Fogel, J. K. J., et al. 2011, ApJ, 734, 98

Öberg, K. I., Furuya, K., Loomis, R., et al. 2015, ApJ, 810, 112

Pascucci, I., Testi, L., Herczeg, G. J., et al. 2016, ApJ, 831, 125

Pinte, C., Dent, W. R. F., Ménard, F., et al. 2016, ApJ, 816, 25

Qi, C., Öberg, K. I., Wilner, D. J., et al. 2013, Science, 341, 630
Qi, C., Öberg, K. I., Andrews, S. M., et al. 2015, ApJ, 813, 128

Reboussin, L., Guilloteau, S., Simon, M., et al. 2015, A\&A, 578, A31

Salinas, V. N., Hogerheijde, M. R., Mathews, G. S., et al. 2017, A\&A, 606, A125

Salter, D. M., Hogerheijde, M. R., van der Burg, R. F. J., Kristensen, L. E., \& Brinch, C. 2011, A\&A, 536, A80

Schwarz, G. 1978, Ann. Stat., 6, 461

Siess, L., Dufour, E., \& Forestini, M. 2000, A\&A, 358, 593

Tazzari, M., Testi, L., Natta, A., et al. 2017, A\&A, 606, A88

Teague, R., Guilloteau, S., Semenov, D., et al. 2016, A\&A, 592, A49

Teague, R., Semenov, D., Gorti, U., et al. 2017, ApJ, 835, 228

Thi, W.-F., van Zadelhoff, G.-J., \& van Dishoeck, E. F. 2004, A\&A, 425, 955

van Kempen, T. A., van Dishoeck, E. F., Brinch, C., \& Hogerheijde, M. R. 2007, A\&A, 461, 983

van der Marel, N., van Dishoeck, E. F., Bruderer, S., et al. 2016a, A\&A, 585, A58

van der Marel, N., Verhaar, B. W., van Terwisga, S., et al. 2016b, A\&A, 592, A126

van der Marel, N., Williams, J. P., Ansdell, M., et al. 2018, ApJ, 854, 177

van Terwisga, S. E., van Dishoeck, E. F., Ansdell, M., et al. 2018, A\&A, 616, A88

van’t Hoff, M. L. R., Walsh, C., Kama, M., Facchini, S., \& van Dishoeck, E. F. 2017, A\&A, 599, A101

Visser, R., Bruderer, S., Cazzoletti, P., et al. 2018, A\&A, 615, A75

Weingartner, J. C., \& Draine, B. T. 2001, ApJ, 548, 296

Woitke, P., Min, M., Thi, W. F., et al. 2018, A\&A, 618, A57

Zhang, K., Bergin, E. A., Blake, G. A., et al. 2016, ApJ, 818, L16 


\section{Appendix A: $\mathrm{CN}$ versus $\mathrm{C}^{18} \mathrm{O}$ in Lupus disks}

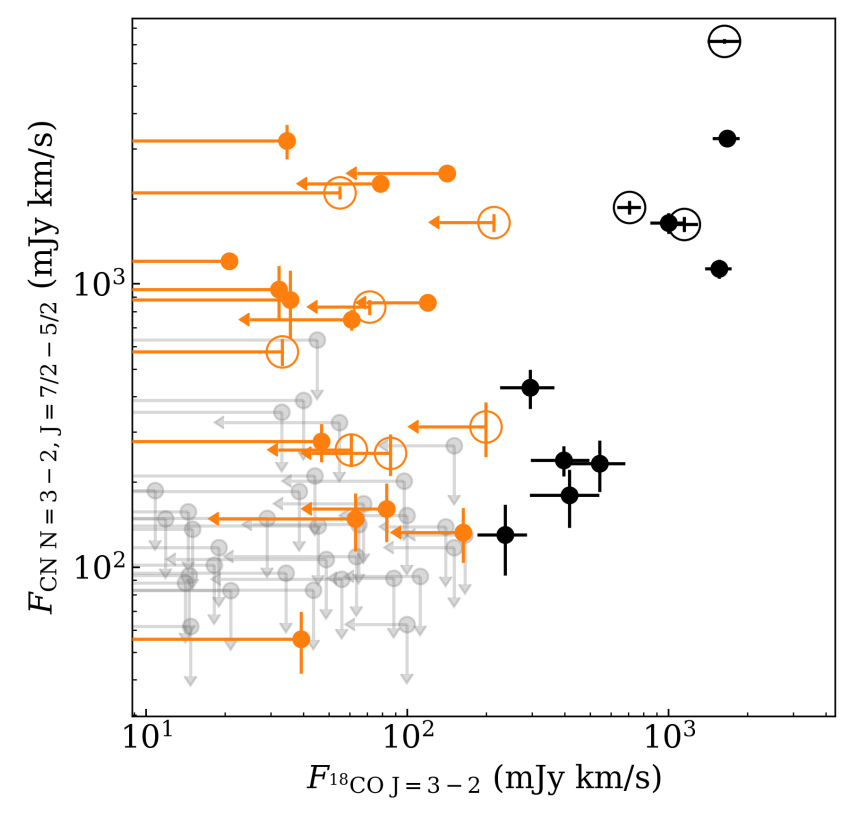

Fig. A.1. Integrated $\mathrm{CN}$ vs. $\mathrm{C}^{18} \mathrm{O} 3-2$ fluxes for the Lupus disk sample. Detected sources are black, $\mathrm{C}^{18} \mathrm{O} 3-2$ upper limits are orange, $\mathrm{CN}$ flux upper limits are blue, and double upper limits are gray. Transition disks are marked with a circle.

\section{Appendix B: $u v$-plane analysis}

It is possible that, in the disks in which a clear $\mathrm{CN}$ ring is present, some underlying continuum structure is responsible, for instance, by causing a local density enhancement. Therefore, it is relevant to first establish if any such structures are present in the disks we study in detail, and if so, where they are located.

In the two disks discussed here, based on their continuum intensity profile (Fig. 6), Sz 98 seems to have a bright central core, with a large, fainter outer region, giving it something of a "fried-egg"-appearance similar to V1094 Sco (van Terwisga et al. 2018); the radial profile seems to suggest an unresolved structure in this outer region. Sz 71, on the other hand, may have a "knee" in its intensity profile at around 50 AU. Analyzing the continuum emission in the $u v$-plane can provide a more thorough picture of the mm-dust structure underlying the $\mathrm{CN}$ emission, even if such features are difficult to detect in the image plane due to the deconvolution algorithm. Tazzari et al. (2017) performed a fit of both disks with a smooth self-similar disk model, including radiative transfer. However, such a smooth model is not expected to be able to fit radial substructures in the continuum, if any are present. For that reason, a simpler fitting method is used here, focusing only on reproducing the radial intensity profile.

Both sources were deprojected and derotated based on an image-plane fit with the CASA imfit-task. We confirmed that, within the uncertainties given by these values, the results of our analysis did not significantly change. Subsequently the data were binned to $15 \mathrm{k} \lambda$ bins in $u v$-space, and finally scaled and fit with two simple models: a Gaussian core (Model 1), the single-component model, and a Gaussian core modulated with a cosinusoidal term (Model 2), the two-component model, to mimic the behavior of an unresolved ring of particles. This method was successfully used by Zhang et al. (2016) to describe rings in a variety of transitional and full disks, and has the important property that it uses a minimal number of parameters, while being smooth (making it similar to these disks' appearance) and not necessarily containing nulls (which must occur for sharp-edged models).

$$
\begin{aligned}
I(\theta)= & \frac{1}{\sqrt{2 \pi} \sigma_{0}} \exp \left(-\frac{\theta^{2}}{2 \sigma_{0}^{2}}\right) \\
& +\sum_{j} \cos \left(2 \pi \theta \rho_{j}\right) \times \frac{a_{j}}{\sqrt{2 \pi} \sigma_{j}} \exp \left(-\frac{\theta^{2}}{2 \sigma_{j}^{2}}\right) .
\end{aligned}
$$

Here, $a_{j}$ is the relative intensity of the $j$ th component, $\sigma_{j}$ its width (converted, for ease of reading, to $\mathrm{AU}$ in Table B.1), $\rho_{j}$ is the spatial frequency which modulates the $j$ th Gaussian, and $\theta$ is the angle to the source center (in radians). Baselines up to $800 \mathrm{k} \lambda$ are used for our fit to $\mathrm{Sz} 71$, and up to $500 \mathrm{k} \lambda$ for $\mathrm{Sz}$ 98; beyond these values, noise starts dominating our $u v$-plane data. Parameter values for two models, with $j_{\max }=0$ and $j_{\max }=1$, were obtained from MCMC-fits to the observations with the PyMC Python module.

In Fig. B.1 and Table B.1, the results of the fitting procedure are shown. For both $\mathrm{Sz} 71$ and $\mathrm{Sz} 98$, the two-component Gaussian model fits the continuum significantly better than the single-component one, when comparing the Bayesian Information Criterion (BIC; Schwarz 1978): $\Delta_{\mathrm{BIC}}=1296.9$ for Sz 71, and for $\mathrm{Sz} 98, \Delta_{\mathrm{BIC}}=1382.7$ in favor of the two-component model. The two-component model performs especially well at large baselines, suggesting that there is continuum structure at small spatial scales. For Sz 98, a true second maximum is immediately obvious in the visibility plane. The similarity of this feature to those seen (at much higher resolutions and sensitivities) in, for example, Zhang et al. (2016), ALMA Partnership (2015), and Andrews et al. (2016), is remarkable, all the more so considering its amplitude - not much fainter than the structures identified in the V1094 Sco disk (van Terwisga et al. 2018). For Sz 71 the bestfit values of $\rho_{1}$ show a fairly large uncertainty, and the amplitude of the small-scale signal is not very large.

In $\mathrm{Sz} 98$, we can assign the identified $u v$-plane feature to the presence of an (unresolved) ring in the image plane, located at $117 \mathrm{AU}$, depicted with a dotted gray line in Fig. 6 (right); this is consistent with the knee that is tentatively identified in the continuum radial intensity profile. Moreover, Tazzari et al. (2017) find a clear ring-like residual when comparing their bestfit model of $\mathrm{Sz} 98$ to the data, located in the same part of the disk. The visibility feature in Sz 71 is weaker and overlaps with the bright "core" of the disk; thus, without higher-resolution and deeper data, we can only say that its brightness profile drops off less steeply beyond $50 \mathrm{AU}$ in this disk (Fig. 6; left). Given the similarity of these disks' visibilities, it is possible that a structure similar to that of $\mathrm{Sz} 98$ is present in $\mathrm{Sz} 71$, but at a fainter level and closer to the central star. However, no ring-like residual is present for this disk in the best-fit smooth disk model by Tazzari et al. (2017). It is also important to note that our intensity profiles do not provide any information on the vertical structure of the continuum, since the mm-sized grains responsible for the emission modeled here are expected to be vertically settled with respect to the disk gas (Dullemond \& Dominik 2005; Pinte et al. 2016). 

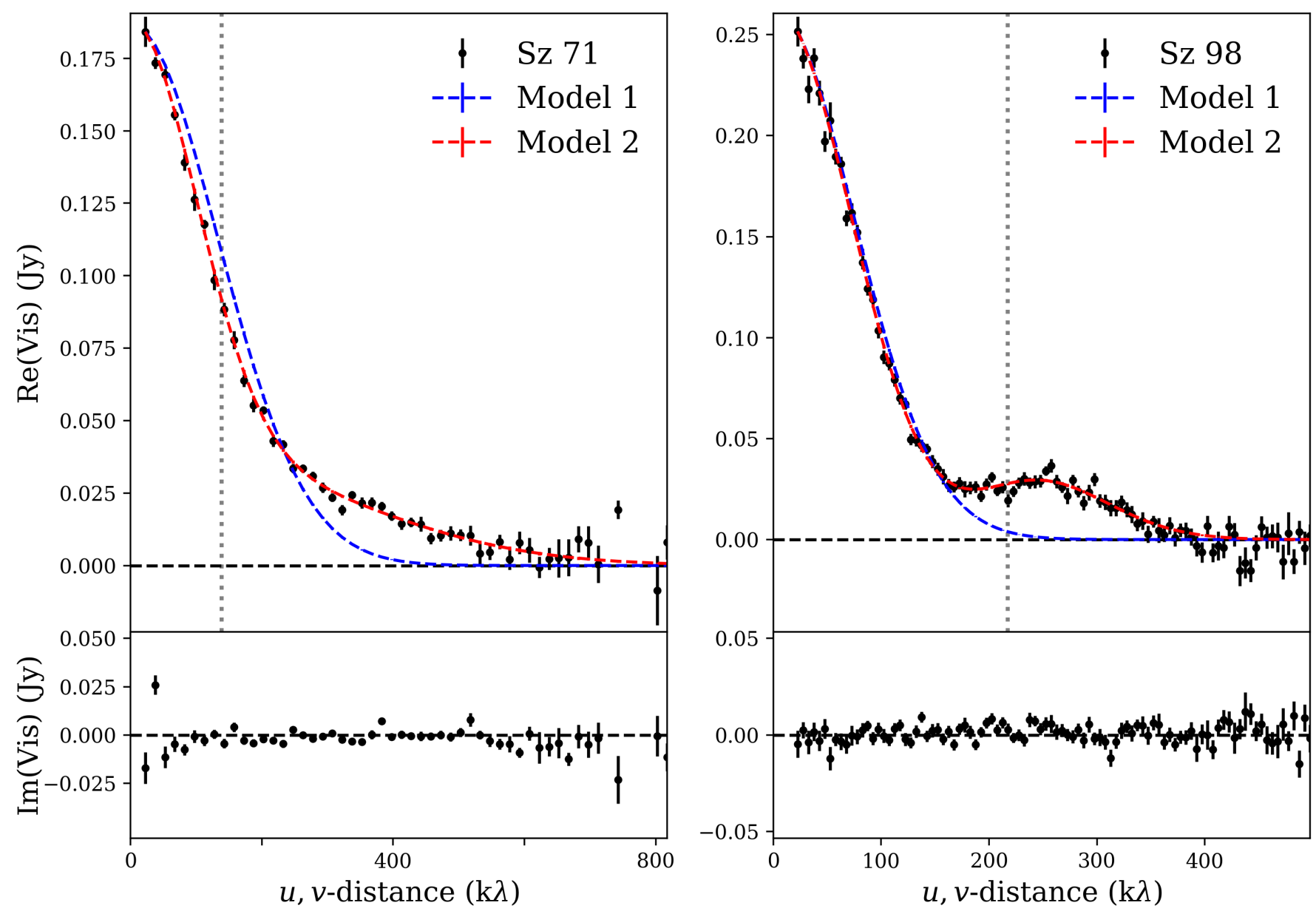

Fig. B.1. $345 \mathrm{GHz}$ continuum visibilities for $\mathrm{Sz} 71$ and $\mathrm{Sz} 98$, after deprojection and derotation. The real visibilities have been overplotted with the best fitted single-component model (Model 1, blue) and the best-fit two-component model (Model 2, red). The best-fit spatial frequency of the two-component model is indicated by the gray dashed lines.

Table B.1. Parameter values and inferred errors from the MCMC fit for both models and sources.

\begin{tabular}{|c|c|c|c|c|c|c|c|}
\hline & & $\begin{array}{l}\sigma_{0} \\
(\mathrm{AU})\end{array}$ & $\begin{array}{l}a_{1} \\
\left(\mathrm{Jy} \mathrm{beam}^{-1}\right)\end{array}$ & $\begin{array}{l}\sigma_{1} \\
(\mathrm{AU})\end{array}$ & $\begin{array}{l}\rho_{1} \\
(\mathrm{k} \lambda) \\
\end{array}$ & $\chi_{v}^{2}$ & BIC \\
\hline Sz 71 & $\begin{array}{l}\text { Model } 1 \\
\text { Model } 2 \\
\end{array}$ & $\begin{array}{l}37.1 \pm 0.18 \\
49.8 \pm 0.80 \\
\end{array}$ & $\overline{-} \mathbf{0} 969 \pm 0.055$ & $\begin{array}{l}- \\
19.9 \pm 1.7\end{array}$ & $\begin{array}{l}- \\
138 \pm 23\end{array}$ & $\begin{array}{l}27.87 \\
1.71 \\
\end{array}$ & $\begin{array}{l}1071.2 \\
-225.7 \\
\end{array}$ \\
\hline Sz 98 & $\begin{array}{l}\text { Model } 1 \\
\text { Model } 2\end{array}$ & $\begin{array}{l}87.7 \pm 0.40 \\
85.3 \pm 0.71\end{array}$ & $\overline{-}-467 \pm 0.11$ & $-\overline{91.0} \pm 3.0$ & $-\overline{217.0 \pm 2.1}$ & $\begin{array}{l}20.67 \\
1.45\end{array}$ & $\begin{array}{l}1306.8 \\
-75.9\end{array}$ \\
\hline
\end{tabular}

\section{Appendix C: SED fitting procedure}

We attempted to closely reproduce the SEDs of both sources with our models, in order to find a dust density and temperature structure corresponding as closely as possible to the real (disk-averaged) properties of the disks, especially at mid-infrared down to mm wavelengths. Using the resulting parametrized disks as input for the DALI physical-chemical modeling code allows us to confirm if the $\mathrm{CN}$ rings we observe in the sources are simply the result of the global properties of the disk (particularly, the interplay between gas density and UV radiation) or if they require underlying dust and/or gas substructures at the radius of the $\mathrm{CN}$ ring (neither of which would be revealed by the SED).

The disk model used is adapted from the model used in Andrews et al. (2011), but without an exponential taper at characteristic radius $R_{\mathrm{c}}$. Instead, the disk is truncated at $R_{\mathrm{c}}=R_{\text {out }}$, and the surface density slope is fixed at $\gamma=1$ for all models. The parameter space of this model was explored in two phases. For the first phase, we used the RADMC3D-code ${ }^{1}$ to globally constrain the flaring angle of the disk $\psi$, its scale height $h_{\mathrm{r}}$, the disk mass $M_{\mathrm{d}}$, and the possibility of an inner cavity $\left(R_{\text {in }} \geq R_{\text {sub,silicate }}\right)$.

Taking $R_{\text {in }}$ as a variable was motivated by our difficulty reproducing the near- and mid-IR parts of the SED when using the standard parametrization of $R_{\text {sub,silicate. However, we only take }}$ values of $R_{\text {in }}$ in a small interval, as radii beyond $\sim 2$ AU would lead to obviously different SEDs. The shape of the near- and mid-IR SED is also sensitive to the choice of parametrization of the inner region structure: a puffed-up inner rim extending to the sublimation radius, for instance, could lead to a similar SED.

\footnotetext{
1 http://www.ita.uni-heidelberg.de/ dullemond/ software/radmc-3d/
} 

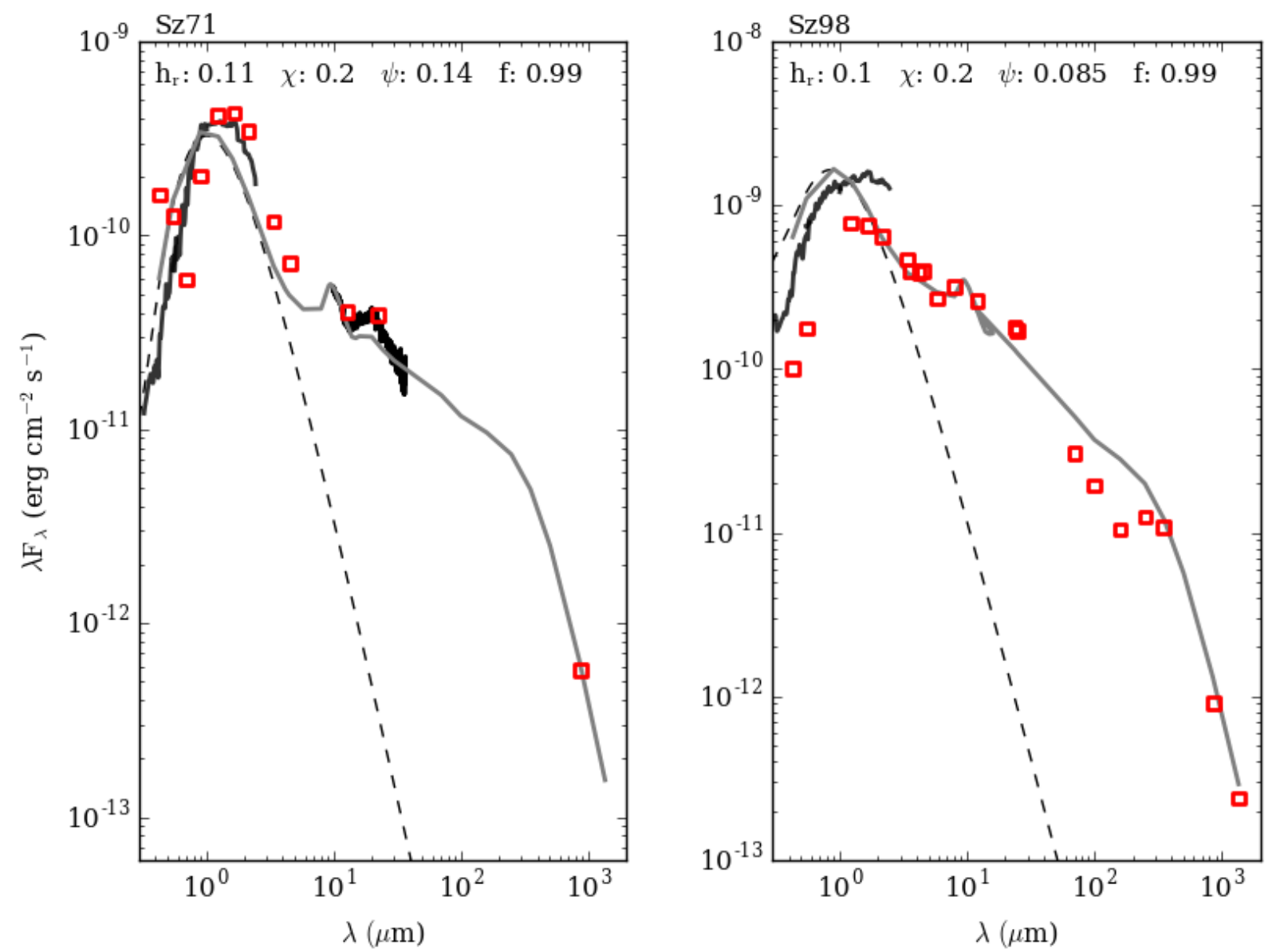

Fig. C.1. DALI SEDs and model parameters for the fiducial disk models. The preferred model is in gray, X-shooter and IRS spectra are solid black lines, broadband continuum data are open red symbols, and the black dashed lines the input stellar photospheres.

However, such changes would make a significant difference only at scales much smaller than our ALMA observations' resolution.

The values of $R_{\text {out }}$ and $i$ in our models are taken from the $870 \mu \mathrm{m} \mathrm{CN}$ and continuum images, respectively. For both disks, we take $R_{\text {out }}$ to be $\sim 200$ AU. We use a single grain population between 0.5 and $1000 \mu \mathrm{m}$. Our opacities are based on the same abundances of amorphous and crystalline silicate and amorphous carbon grains as in Weingartner \& Draine (2001).

The second phase, performed in DALI, uses the best-fit values from the RADMC3D models, but includes a population of small grains and settled large grains. The fraction of large grains, here, is $f$, and the decreased scale height of the settled large grain population is equal to $\chi h_{\mathrm{r}}$. We explore only $f=[0.85,0.99]$ and $\chi=[0.2,1.0]$. Our small grain population is limited to a maximum size of $1 \mu \mathrm{m}$. Including this small grain population means we have a better description of the silicate features in the NIR (especially for $\mathrm{Sz} 71$, for which we have an IRS spectrum). Using DALI also allows us to make sure no gridding inconsistencies are present when we run the full thermo-chemical code to calculate the $\mathrm{CN}$ emission. The resulting models are shown in Fig. C.1.

For the stellar luminosity, effective temperatures, and accretion luminosities we used the values given in Table 1 . We corrected the data for extinction using $R_{\mathrm{V}}=3.1$, and $A_{\mathrm{V}}=0.5 \mathrm{mag}$ and $1.0 \mathrm{mag}$ for $\mathrm{Sz} 71$ and $\mathrm{Sz} 98$, respectively (Alcalá et al. 2017). In the case of $\mathrm{Sz} 71$, the $\mathrm{X}$-shooter spectra have been rescaled by a factor of 1.5 (within the instrument's systematic error; see e.g., Alcalá et al. 2014) to agree with the 2MASS J, $\mathrm{H}, \mathrm{K}$-magnitudes. For $\mathrm{Sz} 98$, we show the $2 \mathrm{MASS}$ and $B$ and
$V$-band photometry, but do not include them in our fit. This source is a known variable, and the $\mathrm{X}$-shooter data have the largest amount of wavelength coverage in a single observation. Moreover, the most accurate determination of $T_{\text {eff }}$ and $L_{\star}$ is based on these data.

\section{C.1. SED data}

The SEDs of the disks were constructed on the basis of available literature data. For Sz 98, the dataset consists of data from the X-shooter (from Alcalá et al. 2017), WISE, and the Herschel PACS and SPIRE instruments (Bustamante et al. 2015), as well as 2MASS $J, H, K$ and $B$ and $V$-band fluxes (Cutri et al. 2003). The PACS data, however, were not used in the fit since they appear to be affected by a systematic offset possibly caused by the photometric calibration.

Sz 71 has not been observed by Herschel, and the behavior of its SED is therefore much less constrained at far-IR wavelengths. However, $B, V, R$-band and X-shooter (from Alcalá et al. 2014) and 2MASS data do exist, as well as a Spitzer IRS spectrum between 10 and $36 \mu \mathrm{m}$ (Lebouteiller et al. 2015). The Sz 71 SED appears to be showing a significant contribution from amorphous carbonaceous grains, in the form of excess emission at wavelengths down to $1 \mu \mathrm{m}$. These opacities are not included in our models. However, since their primary effect is to heat the innermost part of the disk (outside our chemical region of interest) we do not expect to seriously underestimate the $\mathrm{CN}$ emission by excluding them in our full thermo-chemical models. 


\section{C.2. SED fitting results}

Both disks appear to be nonflaring compared to, for example, the sample presented in van der Marel et al. (2016b) when fit with a similar methodology and disk model. This is significant, since the flaring angle has a large impact on the dust heating at radii where we expect $\mathrm{CN}$ to be present (Cazzoletti et al. 2018). However, there is still a significant difference between both disks' flaring angles, meaning our results are still representative of disks in general with a variety of flaring angles.
Table C.1. Parameter ranges and used values for the disk models.

\begin{tabular}{lccc}
\hline \hline Sz71 & & & \\
Parameter & Minimum & Maximum & Used \\
\hline$h_{\mathrm{r}}$ & 0.07 & 0.15 & 0.11 \\
$\psi$ & 0.1 & 0.3 & 0.14 \\
$f$ & 0.85 & 0.99 & 0.99 \\
$\chi$ & 0.2 & 1.0 & 0.2 \\
$R_{\text {in }}(\mathrm{AU})$ & 0.04 & 1.5 & 1.0 \\
$M_{\mathrm{d}}\left(M_{\odot}\right)$ & 0.01 & 0.09 & 0.03 \\
\hline Sz 98 & & & \\
Parameter & Minimum & Maximum & Best \\
\hline$h_{\mathrm{r}}$ & 0.07 & 0.15 & 0.1 \\
$\psi$ & 0.07 & 0.1 & 0.085 \\
$f$ & 0.85 & 0.99 & 0.99 \\
$\chi$ & 0.2 & 1.0 & 0.2 \\
$R_{\text {in }}(\mathrm{AU})$ & 0.06 & 1.5 & 0.5 \\
$M_{\mathrm{d}}\left(M_{\odot}\right)$ & 0.01 & 0.09 & 0.07 \\
\hline
\end{tabular}




\section{Appendix D: CN channel maps and emission line spectra for two bright, resolved sources}

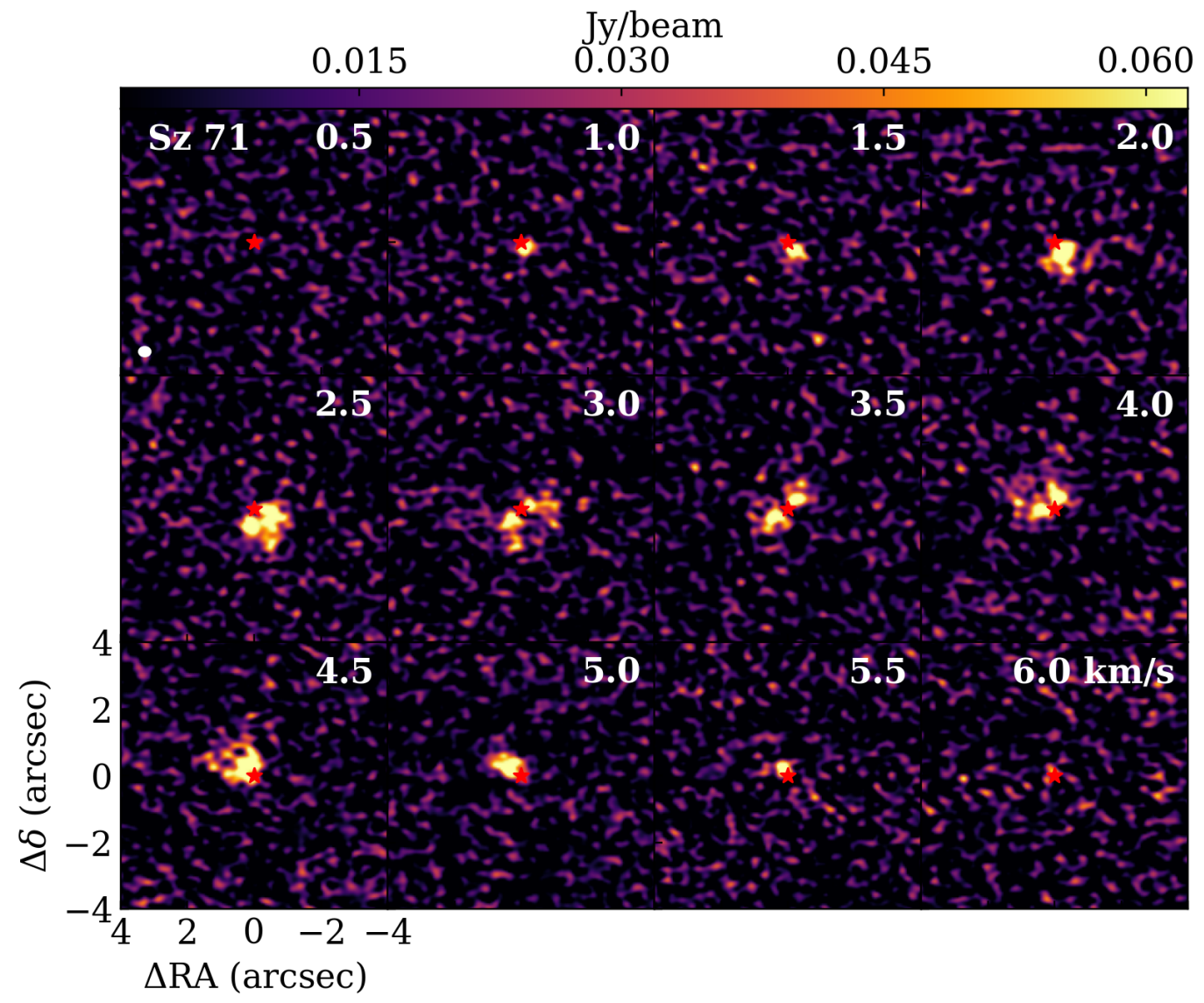

Fig. D.1. Channel map showing $\mathrm{CN}$ emission towards $\mathrm{Sz} 71$, and relatively faint line wings indicative of an emission ring. The source location is marked with a red star; the contours are at the $3 \sigma$ and $5 \sigma$ levels.

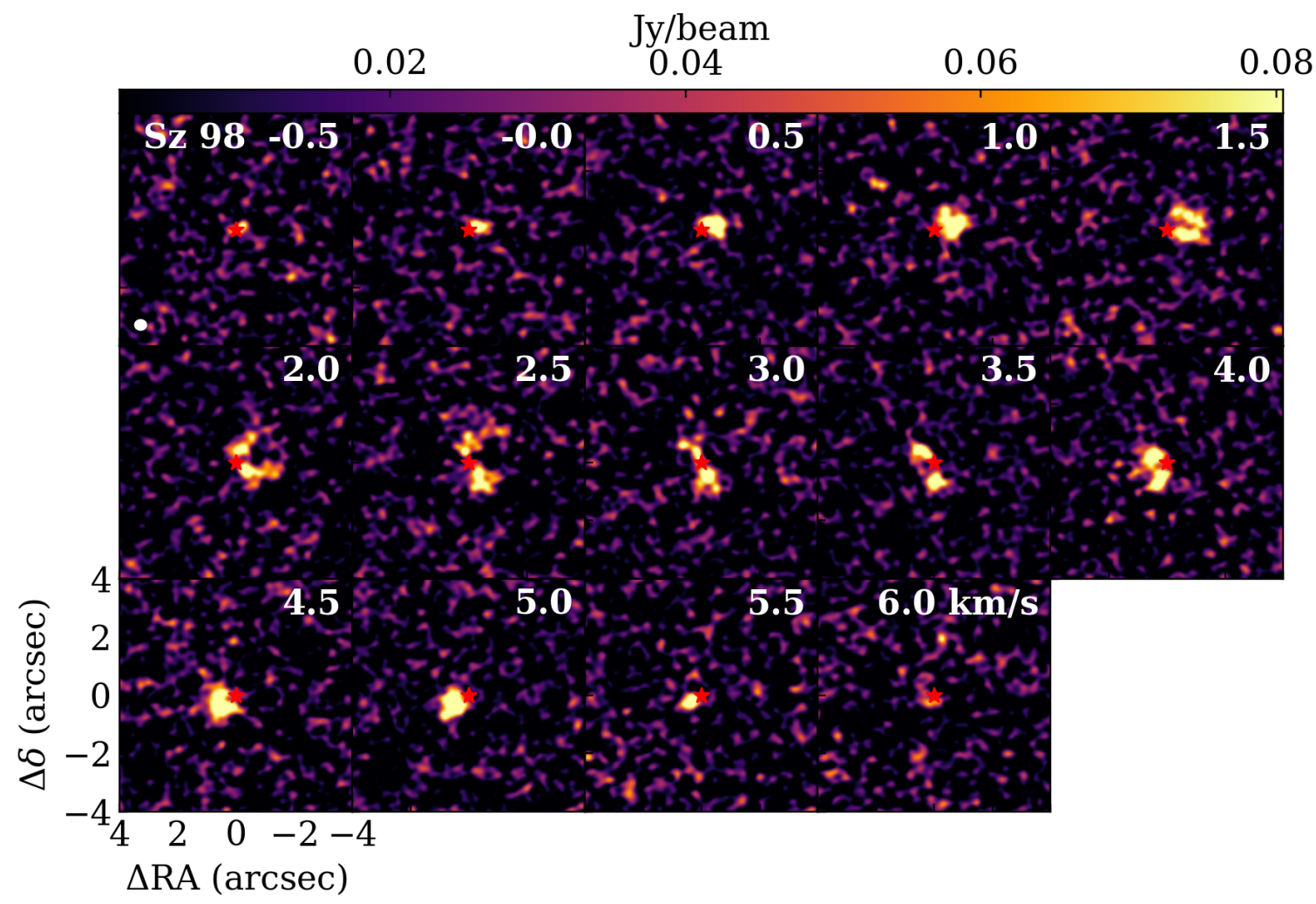

Fig. D.2. Channel map showing $\mathrm{CN}$ emission towards Sz 98, and relatively faint line wings indicative of an emission ring. The source location is marked with a red star; the contours are at the $3 \sigma$ and $5 \sigma$ levels. 

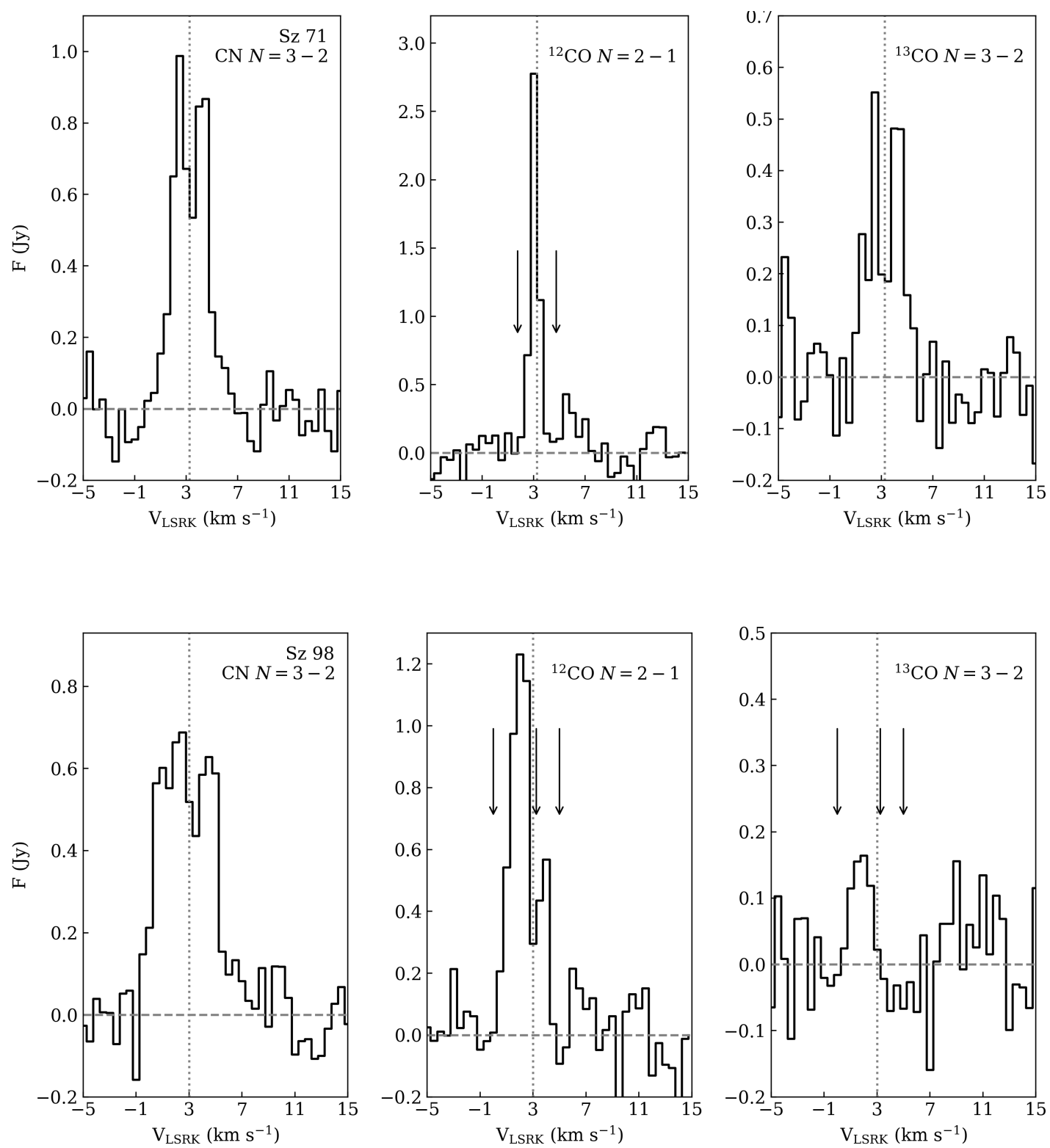

Fig. D.3. Spectra of CN (focusing on the brightest $N=3-2, J=7 / 2-5 / 2$ components) and the main CO isotopologs for Sz 71 (top panels) and Sz 98 (bottom panels). Arrows show the velocities of foreground cloud material (partially) absorbing the ${ }^{12} \mathrm{CO}$ line. Vertical dotted lines indicate the source velocity. The scale of the $y$-axis varies between panels. All spectra were extracted in an aperture overlapping with the detection in the moment-zero map (for ${ }^{12} \mathrm{CO}$ ), or in a $2^{\prime \prime}$ aperture on the source location (for $\mathrm{CN}$ and ${ }^{13} \mathrm{CO}$ ). 
S. E. van Terwisga et al.: The ALMA Lupus protoplanetary disk survey: CN

Appendix E: CN fluxes and upper limits for the Lupus sample

Table E.1. CN $N=3-2, J=7 / 2-5 / 2$ fluxes, errors, and apertures.

\begin{tabular}{|c|c|c|c|}
\hline Source & $\begin{array}{l}F_{\mathrm{CN} N=3-2} \\
\left(\mathrm{Jy} \mathrm{km} \mathrm{s}^{-1}\right)\end{array}$ & $\begin{array}{l}E_{\mathrm{CN} N=3-2} \\
\left(\mathrm{Jy} \mathrm{km} \mathrm{s}^{-1}\right)\end{array}$ & $\begin{array}{l}\text { Aperture } \\
(\operatorname{arcsec})\end{array}$ \\
\hline $\mathrm{Sz} 65$ & 250 & 31 & 0.58 \\
\hline Sz 66 & 57 & 14 & 0.29 \\
\hline J15430131-3409153 & $<635$ & $\ldots$ & 0.35 \\
\hline J15430227-3444059 & $<133$ & $\ldots$ & 0.35 \\
\hline J15445789-3423392 & $<180$ & $\ldots$ & 0.35 \\
\hline J15450634-3417378 & $<139$ & $\ldots$ & 0.35 \\
\hline J15450887-3417333 & 245 & 51 & 0.58 \\
\hline Sz 68 & 191 & 44 & 0.58 \\
\hline $\mathrm{Sz} 69$ & $<344$ & $\ldots$ & 0.35 \\
\hline $\mathrm{Sz} 71$ & 2353 & 95 & 1.45 \\
\hline $\mathrm{Sz} 72$ & $<95$ & $\ldots$ & 0.35 \\
\hline $\mathrm{Sz} 73$ & $<121$ & $\begin{array}{l}\cdots \\
\cdots\end{array}$ & 0.35 \\
\hline $\mathrm{Sz} 74$ & $<111$ & $\ldots$ & 0.35 \\
\hline $\mathrm{Sz} 75$ & $<69$ & $\ldots$ & 0.35 \\
\hline $\mathrm{Sz} 76$ & 949 & 202 & 1.62 \\
\hline Sz 77 & $<124$ & $\ldots$ & 0.35 \\
\hline $\mathrm{Sz} 81 \mathrm{~A}$ & $<166$ & $\ldots$ & 0.35 \\
\hline $\mathrm{J} 15560210-3655282$ & 890 & 238 & 1.16 \\
\hline $\mathrm{IM} \mathrm{Lup}^{a}$ & 3289 & 130 & \\
\hline $\mathrm{Sz} 83$ & 1630 & 135 & 1.45 \\
\hline Sz 84 & 626 & 68 & 1.16 \\
\hline $\mathrm{Sz} 129$ & 724 & 61 & 1.16 \\
\hline J15592523-4235066 & $<109$ & $\ldots$ & 0.35 \\
\hline RY Lup & 1624 & 100 & 1.16 \\
\hline J16000060-4221567 & $<106$ & $\ldots$ & 0.35 \\
\hline J16000236-4222145 & 1983 & 114 & 2.03 \\
\hline J16002612-4153553 & $<108$ & $\ldots$ & 0.35 \\
\hline Sz 130 & $<140$ & $\ldots$ & 0.35 \\
\hline MY Lup & 1700 & 123 & 1.45 \\
\hline Sz 131 & $<89$ & $\ldots$ & 0.35 \\
\hline J16011549-4152351 & 1131 & 86 & 1.16 \\
\hline EX Lup & $<63$ & $\ldots$ & 0.35 \\
\hline Sz 133 & 2655 & 112 & 1.16 \\
\hline Sz 88A & $<243$ & $\ldots$ & 0.35 \\
\hline $\mathrm{Sz} 88 \mathrm{~B}$ & $<95$ & $\ldots$ & 0.35 \\
\hline J16070384-3911113 & 430 & 67 & 1.16 \\
\hline J16070854-3914075 & 1719 & 91 & 1.16 \\
\hline $\mathrm{Sz} 90$ & 273 & 42 & 0.58 \\
\hline Sz 91 & $\ldots$ & $\ldots$ & \\
\hline J16073773-3921388 & 50 & 13 & 0.29 \\
\hline Sz 95 & $<94$ & $\ldots$ & 0.35 \\
\hline J16075475-3915446 & $<388$ & $\ldots$ & 0.35 \\
\hline J16080017-3902595 & $<183$ & $\ldots$ & 0.35 \\
\hline J16080175-3912316 & $<202$ & $\ldots$ & 0.35 \\
\hline Sz 96 & $<145$ & $\ldots$ & 0.35 \\
\hline J16081497-3857145 & 160 & 37 & 0.58 \\
\hline Sz 97 & $<152$ & $\ldots$ & 0.35 \\
\hline $\mathrm{Sz} 98$ & 3494 & 131 & 1.74 \\
\hline Sz 99 & $<149$ & $\ldots$ & 0.35 \\
\hline Sz 100 & 350 & 45 & 0.87 \\
\hline J160828.1-391310 & $<235$ & $\ldots$ & 0.35 \\
\hline Sz 102 & $<83$ & $\ldots$ & 0.35 \\
\hline
\end{tabular}

Notes. ${ }^{(a)} \mathrm{CN} N=2-1, J=5 / 2-3 / 2$ flux scaled to the $\mathrm{CN} N=$ $3-2, J=7 / 2-5 / 2$ transition. ${ }^{(b)}$ Partly resolved-out and continuumshielded.
Table E.1. continued.

\begin{tabular}{|c|c|c|c|}
\hline Source & $\begin{array}{l}F_{\mathrm{CN} N=3-2} \\
\left(\mathrm{Jy} \mathrm{km} \mathrm{s}^{-1}\right)\end{array}$ & $\begin{array}{l}E_{\mathrm{CN} N=3-2} \\
\left(\mathrm{Jy} \mathrm{km} \mathrm{s}^{-1}\right)\end{array}$ & $\begin{array}{l}\text { Aperture } \\
\text { (arcsec) }\end{array}$ \\
\hline Sz 103 & $<258$ & $\ldots$ & 0.35 \\
\hline $\mathrm{J} 16083070-3828268$ & 7467 & 134 & 2.03 \\
\hline Sz 104 & $<105$ & $\ldots$ & 0.35 \\
\hline $\mathrm{J} 160831.1-385600$ & $<132$ & $\ldots$ & 0.35 \\
\hline V856 Sco & $<205$ & $\ldots$ & 0.35 \\
\hline V1094 $\mathrm{Sco}^{b}$ & 3430 & 475 & 5.34 \\
\hline Sz 106 & $<152$ & $\ldots$ & 0.35 \\
\hline Sz 108B & 115 & 32 & 0.58 \\
\hline J16084940-3905393 & $<98$ & $\ldots$ & 0.35 \\
\hline V1192 Sco & $<112$ & $\ldots$ & 0.35 \\
\hline Sz 110 & $<95$ & $\ldots$ & 0.35 \\
\hline J16085324-3914401 & 119 & 26 & 0.46 \\
\hline J16085373-3914367 & 182 & 38 & 0.58 \\
\hline Sz 111 & 1881 & 106 & 2.03 \\
\hline J16085529-3848481 & $<190$ & $\ldots$ & 0.35 \\
\hline Sz 112 & $<82$ & $\ldots$ & 0.35 \\
\hline Sz 113 & 140 & 33 & 0.58 \\
\hline J16085828-3907355 & $<353$ & $\ldots$ & 0.35 \\
\hline J16085834-3907491 & $<126$ & $\ldots$ & 0.35 \\
\hline J16090141-3925119 & 805 & 47 & 0.87 \\
\hline $\mathrm{Sz} 114$ & 1158 & 55 & 1.16 \\
\hline Sz 115 & $<150$ & $\ldots$ & 0.35 \\
\hline J16091644-3904438 & $<272$ & $\ldots$ & 0.35 \\
\hline $\mathrm{J} 16092032-3904015$ & $<277$ & $\ldots$ & 0.35 \\
\hline J16092317-3904074 & $<136$ & $\ldots$ & 0.35 \\
\hline J16092697-3836269 & $<268$ & $\cdots$ & 0.35 \\
\hline J160934.2-391513 & $<135$ & $\ldots$ & 0.35 \\
\hline J16093928-3904316 & $<281$ & $\ldots$ & 0.35 \\
\hline Sz 117 & $<153$ & $\ldots$ & 0.35 \\
\hline $\mathrm{Sz} 118$ & 295 & 65 & 0.87 \\
\hline J16095628-3859518 & $<95$ & $\ldots$ & 0.35 \\
\hline J16100133-3906449 & $<132$ & $\ldots$ & 0.35 \\
\hline J16101307-3846165 & $<106$ & $\ldots$ & 0.35 \\
\hline J16101857-3836125 & $<107$ & $\ldots$ & 0.35 \\
\hline J16101984-3836065 & $<89$ & $\ldots$ & 0.35 \\
\hline J16102741-3902299 & $<130$ & $\ldots$ & 0.35 \\
\hline J16102955-3922144 & 787 & 48 & 0.87 \\
\hline $\mathrm{Sz} 123 \mathrm{~B}$ & $<102$ & $\ldots$ & 0.35 \\
\hline $\mathrm{Sz} 123 \mathrm{~A}$ & 241 & 41 & 0.64 \\
\hline J16115979-3823383 & $<95$ & $\ldots$ & 0.35 \\
\hline J16120445-3809589 & $<219$ & $\ldots$ & 0.35 \\
\hline J16124373-3815031 & 1015 & 82 & 0.99 \\
\hline J16134410-3736462 & $<149$ & $\ldots$ & 0.35 \\
\hline
\end{tabular}

